\title{
Stability Notions and Lyapunov Functions for Sliding Mode Control Systems
}

\author{
Andrey Polyakov*1, Leonid Fridman** 2 \\ * - Non-A INRIA - LNE, Parc Scientifique de la Haute Borne 40, avenue Halley Bat.A, \\ Park Plaza 59650 Villeneuve d'Ascq, (e-mail:andrey.polyakov@inria.fr) \\ ** - Departamento de Ingenieria de Control y Robotica, UNAM, Edificio T, Ciudad \\ Universitaria D.F., Mexico, (e-mail: lfridman@servidor.unam.mx)
}

\begin{abstract}
The paper surveys mathematical tools required for stability and convergence analysis of modern sliding mode control systems. Elements of Filippov theory of differential equations with discontinuous right-hand sides and its recent extensions are discussed. Stability notions (from Lyapunov stability (1982) to fixed-time stability (2012)) are observed. Concepts of generalized derivatives and non-smooth Lyapunov functions are considered. The generalized Lyapunov theorems for stability analysis and convergence time estimation are presented and supported by examples from sliding mode control theory.
\end{abstract}

\section{Introduction}

During whole history of control theory, a special interest of researchers was focused on systems with relay and discontinuous (switching) control elements $[1,2,3,4]$. Relay and variable structure control systems have found applications in many engineering areas. They are simple, effective, cheap and sometimes they have better dynamics than linear systems [2]. In practice both input and output of a system may be of a relay type. For example, automobile engine control sys-

\footnotetext{
${ }^{1}$ This work is supported by ANR Grant CHASLIM (ANR-11-BS03-0007).

${ }^{2}$ Grant 190776 Bilateral Cooperation CONACyT(Mexico) - CNRS(France), Grant CONACyT 132125 y Programa de Apoyo a Proyectos de Investiagacion e Inovacion Tecnologico (PAPIIT) 113613 of UNAM.
}

Preprint submitted to Elsevier

December 2, 2013 
tems sometimes use $\lambda$ - sensor with almost relay output characteristics, i.e only the sign of a controllable output can be measured [5]. In the same time, terristors can be considered as relay "actuators" for some power electronic systems $[6]$.

Mathematical backgrounds for a rigorous study of variable structure control systems were presented in the beginning of 1960s by the celebrated Filippov theory of differential equations with discontinuous right-hand sides [7]. Following this theory, discontinuous differential equations have to be extended to differential inclusions. This extension helps to describe, correctly from a mathematical point of view, such a phenomenon as sliding mode [3], [8], [6]. In spite of this, Filippov theory was severely criticized by many authors [9], [10], [3], since it does not describe adequately some discontinuous and relay models. That is why, extensions and specifications of this theory appear rather frequently [10], [11]. Recently, in [12] an extension of Filippov theory was presented in order to study Input-to-State Stability (ISS) and some other robustness properties of discontinuous models.

Analysis of sliding mode systems is usually related to a specific property, which is called finite-time stability [13], [3], [14], [15], [16]. Indeed, the simplest example of a finite-time stable system is the relay sliding mode system: $\dot{x}=-\operatorname{sign}[x], x \in \mathbb{R}, x(0)=x_{0}$. Any solution of this system reaches the origin in a finite time $T\left(x_{0}\right)=\left|x_{0}\right|$ and remains there for all later time instants. Sometimes, this conceptually very simple property is hard to prove theoretically. From a practical point of view, it is also important to estimate a time of stabilization (settling time). Both these problems can be tackled by Lyapunov Function Method [17, 18, 19]. However, designing a finite-time Lyapunov function of a rather simple form is a difficult problem for many sliding mode systems. In particular, appropriate Lyapunov functions for second order sliding mode systems are non-smooth [20, 21, 22] or even non-Lipschitz [23, 24, 25]. Some problems of a stability analysis using generalized Lyapunov functions are studied in [26, 27, 28, 29].

One more extension of a conventional stability property is called fixed-time 
stability [30]. In addition to finite-time stability it assumes uniform boundedness of a settling time on a set of admissible initial conditions (attraction domain). This phenomenon was initially discovered in the context of systems that are homogeneous in the bi-limit [31]. In particular, if an asymptotically stable system has an asymptotically stable homogeneous approximation at the 0-limit with negative degree and an asymptotically stable homogeneous approximation at the $+\infty$-limit with positive degree, then it is fixed-time stable. An important application of this concepts was considered in the paper [32], which designs a uniform (fixed-time) exact differentiator basing on the second order sliding mode technique. Analysis of fixed-time stable sliding mode system requires applying generalized Lyapunov functions [30], [32].

The main goal of this paper is to survey mathematical tools required for stability analysis of modern sliding mode control systems. The paper is organized as follows. The next section presents notations, which are used in the paper. Section 3 considers elements of the theory of differential equations with discontinuous right-hand sides, which are required for a correct description of sliding modes. Stability notions, which frequently appear in sliding mode control systems, are discussed in Section 4. Concepts of generalized derivatives are studied in Section 5 in order to present a generalized Lyapunov function method in Section 6. Finally, some concluding remarks are given.

\section{Notations}

- $\mathbb{R}$ is the set of real numbers and $\overline{\mathbb{R}}=\mathbb{R} \cup\{-\infty\} \cup\{+\infty\}, \mathbb{R}_{+}=\{x \in \mathbb{R}$ : $x>0\}$ and $\overline{\mathbb{R}}_{+}=\mathbb{R}_{+} \cup\{+\infty\}$.

- I denotes one of the following intervals: $[a, b],(a, b),[a, b)$ or $(a, b]$, where $a, b \in \bar{R}, a<b$.

- The inner product of $x, y \in \mathbb{R}^{n}$ is denoted by $\langle x, y\rangle$ and $\|x\|=\sqrt{\langle x, x\rangle}$.

- The set consisting of elements $x_{1}, x_{2}, \ldots, x_{n}$ is denoted by $\left\{x_{1}, x_{2}, \ldots, x_{n}\right\}$.

- The set of all subsets of a set $M \subseteq \mathbb{R}^{n}$ is denoted by $2^{M}$. 
- The sign function is defined by

$$
\operatorname{sign}_{\sigma}[\rho]=\left\{\begin{array}{ccc}
1 & \text { if } & \rho>0, \\
-1 & \text { if } & \rho<0, \\
\sigma & \text { if } & \rho=0,
\end{array}\right.
$$

where $\sigma \in \mathbb{R}:-1 \leq \sigma \leq 1$. If $\sigma=0$ we use the notation $\operatorname{sign}[\rho]$.

- The set-valued modification of the sign function is given by

$$
\overline{\operatorname{sign}}[\rho]=\left\{\begin{array}{ccc}
\{1\} & \text { if } & \rho>0, \\
\{-1\} & \text { if } & \rho<0, \\
{[-1,1]} & \text { if } & \rho=0 .
\end{array}\right.
$$

- $x^{[\alpha]}=|x|^{\alpha} \operatorname{sign}[x]$ is a power operation, which preserves the sign of a number $x \in \mathbb{R}$.

- The geometric sum of two sets is denoted by "++, i.e.

$$
\mathbf{M}_{1} \dot{+} \mathbf{M}_{2}=\bigcup_{x_{1} \in \mathbf{M}_{1}, x_{2} \in \mathbf{M}_{2}}\left\{x_{1}+x_{2}\right\}
$$

where $\mathbf{M}_{1} \subseteq \mathbb{R}^{n}, \mathbf{M}_{2} \subseteq \mathbb{R}^{n}$.

- The Cartesian product of sets is denoted by $\times$.

- The product of a scalar $y \in \mathbb{R}$ and a set $\mathbf{M} \subseteq \mathbb{R}^{n}$ is denoted by "." :

$$
y \cdot \mathbf{M}=\mathbf{M} \cdot y=\bigcup_{x \in \mathbf{M}}\{y x\}
$$

- The product of a matrix $A \in \mathbb{R}^{m \times n}$ to a set $\mathbf{M} \subseteq \mathbb{R}^{n}$ is also denoted by $" . "$

$$
A \cdot \mathbf{M}=\bigcup_{x \in \mathbf{M}}\{A x\} .
$$

- $\partial \Omega$ is the boundary set of $\Omega \subseteq \mathbb{R}^{n}$.

- $\mathcal{B}(r)=\left\{x \in \mathbb{R}^{n}:\|x\|<r\right\}$ is an open ball of the radius $r \in \mathbb{R}_{+}$with the center at the origin. Under introduced notations, $\{y\} \dot{+} \mathcal{B}(\varepsilon)$ is an open ball of the radius $\varepsilon>0$ with the center at $y \in \mathbb{R}^{n}$. 
- $\operatorname{int}(\Omega)$ is the interior of a set $\Omega \subseteq \mathbb{R}^{n}$, i.e. $x \in \operatorname{int}(\Omega)$ iff $\exists r \in \mathbb{R}_{+}$: $\{x\}+\mathcal{B}(r) \subseteq \Omega$.

- Let $k$ be a given natural number. $\mathbb{C}^{k}(\Omega)$ is the set of continuous functions defined on a set $\Omega \subseteq \mathbb{R}^{n}$, which are continuously differentiable up to the order $k$.

- If $V(\cdot) \in \mathbb{C}^{1}$ then $\nabla V(x)=\left(\frac{\partial V}{\partial x_{1}}, \ldots, \frac{\partial V}{\partial x_{n}}\right)^{T}$. If $s: \mathbb{R}^{n} \rightarrow \mathbb{R}^{m}, s(\cdot)=$ $\left(s_{1}(\cdot), \ldots, s_{m}(\cdot)\right)^{T}, s_{i}(\cdot) \in \mathbb{C}^{1}$ then $\nabla s(x)$ is the matrix $\mathbb{R}^{n \times m}$ of the partial derivatives $\frac{\partial s_{j}}{\partial x_{i}}$.

- $\mathbb{W}_{\mathcal{I}}^{n}$ is the set of vector-valued, componentwise locally absolutely continuous functions, which map $\mathcal{I}$ to $\mathbb{R}^{n}$.

\section{Discontinuous systems, sliding modes and disturbances}

\subsection{Systems with discontinuous right-hand sides}

The classical theory of differential equations [33] introduces a solution of the ordinary differential equation (ODE)

$$
\dot{x}=f(t, x), \quad f: \mathbb{R} \times \mathbb{R}^{n} \rightarrow \mathbb{R}^{n},
$$

as a differentiable function $x: \mathbb{R} \rightarrow \mathbb{R}^{n}$, which satisfies (6) on some segment (or interval) $\mathcal{I} \subseteq \mathbb{R}$. The modern control theory frequently deals with dynamic systems, which are modeled by ODE with discontinuous right-hand sides $[6,34$, 35]. The classical definition is not applicable to such ODE. This section observes definitions of solutions for systems with piecewise continuous right-hand sides, which are useful for sliding mode control theory.

Recall that a function $f: \mathbb{R}^{n+1} \rightarrow \mathbb{R}^{n}$ is piece-wise continuous iff $\mathbb{R}^{n+1}$ consists of a finite number of domains (open connected sets) $G_{j} \subset \mathbb{R}^{n+1}, j=$ $1,2, \ldots, N ; G_{i} \bigcap G_{j}=\emptyset$ for $i \neq j$ and the boundary set $\mathcal{S}=\bigcup_{i=1}^{N} \partial G_{j}$ of measure zero such that $f(t, x)$ is continuous in each $G_{j}$ and for each $\left(t^{*}, x^{*}\right) \in \partial G_{j}$ there exists a vector $f^{j}\left(t^{*}, x^{*}\right)$, possible depended on $j$, such that for any sequence 
$\left(t^{k}, x^{k}\right) \in G_{j}:\left(t^{k}, x^{k}\right) \rightarrow\left(t^{*}, x^{*}\right)$ we have $f\left(t^{k}, x^{k}\right) \rightarrow f^{j}\left(t^{*}, x^{*}\right)$. Let functions $f^{j}: \mathbb{R}^{n+1} \rightarrow \mathbb{R}^{n}$ be defined on $\partial G_{j}$ according to this limiting process, i.e.

$$
f^{j}(t, x)=\lim _{\left(t^{k}, x^{k}\right) \rightarrow(t, x)} f\left(t^{k}, x^{k}\right), \quad\left(t^{k}, x^{k}\right) \in G_{j}, \quad(t, x) \in \partial G_{j} .
$$

\subsubsection{Filippov definition}

Introduce the following differential inclusion

$$
K[f](t, x)=\left\{\begin{array}{clc}
\dot{x} \in K[f](t, x), \quad t \in \mathbb{R}, & \\
\operatorname{co}\left(\bigcup_{j \in \mathcal{N}(t, x)}\left\{f^{j}(t, x)\right\}\right) & \text { if } & (t, x) \in \mathbb{R}^{n+1} \backslash \mathcal{S},
\end{array}\right.
$$

where $\operatorname{co}(M)$ is the convex closure of a set $M$ and the set-valued index function $\mathcal{N}: \mathbb{R}^{n+1} \rightarrow 2^{\{1,2, \ldots, N\}}$ defined on $\mathcal{S}$ indicates domains $G_{j}$, which have a common boundary point $(t, x) \in \mathcal{S}$, i.e.

$$
\mathcal{N}(t, x)=\left\{j \in\{1,2, \ldots, N\}:(t, x) \in \partial G_{j}\right\} .
$$

For $(t, x) \in \mathcal{S}$ the set $K[f](t, x)$ is a convex polyhedron.

Definition 1 ([7], page 50). An absolutely continuous function $x: \mathcal{I} \rightarrow \mathbb{R}^{n}$ defined on some interval or segment $\mathcal{I}$ is called a solution of (6) if it satisfies the differential inclusion (7) almost everywhere on $\mathcal{I}$.

Consider the simplest case when the function $f(t, x)$ has discontinuities on a smooth surface $\mathcal{S}=\left\{x \in \mathbb{R}^{n}: s(x)=0\right\}$, which separates $\mathbb{R}^{n}$ on two domains $G^{+}=\left\{x \in \mathbb{R}^{n}: s(x)>0\right\}$ and $G^{-}=\left\{x \in \mathbb{R}^{n}: s(x)<0\right\}$.

Let $P(x)$ be the tangential plane to the surface $\mathcal{S}$ at a point $x \in \mathcal{S}$ and

$$
f^{+}(t, x)=\lim _{x_{i} \rightarrow x, x_{i} \in G^{+}} f\left(t, x_{i}\right) \text { and } f^{-}(t, x)=\lim _{x_{i} \rightarrow x, x_{i} \in G^{-}} f\left(t, x_{i}\right)
$$

For $x \in \mathcal{S}$ the set $K[f](t, x)$ defines a segment connecting the vectors $f^{+}(t, x)$ and $f^{-}(t, x)$ (see Fig. 1(a), 1(b)). If this segment crosses $P(x)$ then the cross point is the end of the velocity vector, which defines the system motion on the 
surface $S$ (see Fig. 1(b)). In this case the system (7) has trajectories, which start to slide on the surface $\mathcal{S}$ according to the sliding motion equation

$$
\dot{x}=f_{0}(t, x),
$$

where the function

$$
f_{0}(t, x)=\frac{\left\langle\nabla s(x), f^{-}(t, x)\right\rangle f^{+}(t, x)+\left\langle\nabla s(x), f^{+}(t, x)\right\rangle f^{-}(t, x)}{\left\langle\nabla s(x), f^{+}(t, x)-f^{-}(t, x)\right\rangle}
$$

is the velocity vector defined by a cross-point of the segment and the plane $P(x)$, i.e. $f_{0}(t, x)=\mu f^{+}(t, x)+(1-\mu) f^{-}(t, x)$ with $\mu \in[0,1]$ such that $\left\langle\nabla s(x), \mu f^{+}(t, x)+(1-\mu) f^{-}(t, x)\right\rangle=0$.

If $\nabla s(x) \not \perp \mu f^{-}(t, x)+(1-\mu) f^{+}(t, x)$ for every $\mu \in[0,1]$ then any trajectory of (7) comes through the surface (see Fig. 1(a)) resulting an isolated "switching" in the right-hand side of (6).

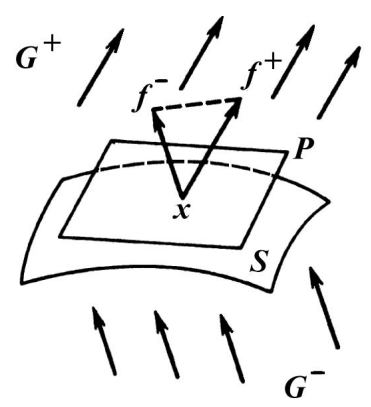

(a) Switching case.

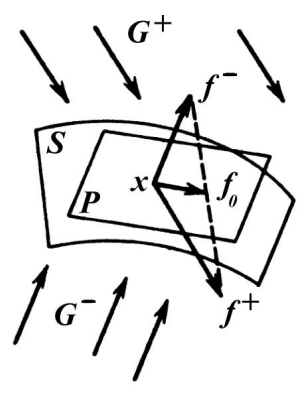

(b) Sliding mode case.

Figure 1: Geometrical illustration of Filippov definition.

Seemingly, Filippov definition is the most simple and widespread definition of solutions for ODE with discontinuous by $x$ right-hand sides. However, this definition was severely criticized by many authors [9], [3], [10] since its appearance in 1960s. In fact, it does not cover correctly many real-life systems, which have discontinuous models. Definitely, contradictions to reality usually are provoked by model inadequacies, but some problems can be avoided by modifications of Filippov definition. 
Example 1. Consider the discontinuous control system

$$
\left\{\begin{array}{l}
\dot{x}_{1}=u, \\
\dot{x}_{2}=\left(\varepsilon u^{2}+\varepsilon^{2}|u|-\varepsilon\right) x_{2}, \quad u=-\operatorname{sign}\left[x_{1}\right],
\end{array}\right.
$$

where $x_{1}, x_{2} \in \mathbb{R}$ are system states, $\varepsilon \in \mathbb{R}_{+}$is some small parameter $0<\varepsilon \ll 1$, $u \in \mathbb{R}$ is the relay control with the sign function defined by (1).

If we apply Filippov definition only to the first equation of (11), we obtain the following sliding motion equation $\dot{x}_{1}=0$ for $x_{1}=0$, which implicitly implies $u=0$ for $x_{1}=0$. So, the expectable sliding motion equation for (11) is

$$
\left\{\begin{array}{l}
\dot{x}_{1}=0, \\
\dot{x}_{2}=-\varepsilon x_{2}, \quad \text { for } \quad x_{1}=0 .
\end{array}\right.
$$

However, considering Filippov definition for the whole system (11) we derive

$$
\begin{aligned}
& f^{+}\left(x_{1}, x_{2}\right)=\left(\begin{array}{c}
-1 \\
\varepsilon^{2} x_{2}
\end{array}\right) \text { for } x_{1} \rightarrow+0 \\
& f^{-}\left(x_{1}, x_{2}\right)=\left(\begin{array}{c}
1 \\
\varepsilon^{2} x_{2}
\end{array}\right) \text { for } x_{1} \rightarrow-0
\end{aligned}
$$

and the formula (10) for $s(x)=x_{1}$ gives another sliding motion equation:

$$
\left(\begin{array}{c}
\dot{x}_{1} \\
\dot{x}_{2}
\end{array}\right)=\frac{\left\langle\nabla s(x), f^{-}(t, x)\right\rangle f^{+}(t, x)+\left\langle\nabla s(x), f^{+}(t, x)\right\rangle f^{-}(t, x)}{\left\langle\nabla s(x), f^{+}(t, x)-f^{-}(t, x)\right\rangle}=\left(\begin{array}{c}
0 \\
\varepsilon^{2} x_{2}
\end{array}\right)
$$

From the practical point of view the sliding motion equation (12) looks more realistic. Indeed, in practice we usually do not have ideal relays, so the model of switchings like (1) is just a "comfortable" approximation of real "relay" elements, which are continuous functions (or singular outputs of additional dynamics [36]) probably with hysteresis or delay effects. In this case, a "real" sliding mode is, in fact, a switching regime of bounded frequency. An average value of the control input

$$
|u|_{\text {average }}=\frac{1}{t-t_{0}} \int_{t_{0}}^{t}|u(\tau)| d \tau, \quad t>t_{0}: x_{1}\left(t_{0}\right)=0
$$

in the "real" sliding mode is less than 1 , particulary $|u|_{\text {average }} \leq 1-\varepsilon$ (see [36] for details). Hence, $\varepsilon|u|_{\text {average }}^{2}+\varepsilon^{2}|u|_{\text {average }}-\varepsilon \leq-\varepsilon^{2}$ and the system (11) has 
asymptotically stable equilibrium point $\left(x_{1}, x_{2}\right)=0 \in \mathbb{R}^{2}$, but Filippov definition quite the contrary provides instability of the system.

Such problems with Filippov definition may appear if the control input $u$ is incorporated to the system (11) in nonlinear way. More detailed study of such discontinuous models is presented in [11].

This example demonstrates two important things:

- Filippov defintion is not appropriate for some discontinuous models, since it does not describe a real system motion.

- Stability properties of a system with discontinuous right-hand side may depend on a definition of solutions.

Remark 1 (On Filippov regularization). The regularization of the ODE system with discontinuous right-hand side can be also done even if the function $f(t, x)$ in (6) is not piecewise continuous, but locally measurable. In this case the differential inclusion (7) has the following right-hand side [7]:

$$
K[f](t, x)=\bigcap_{\delta>0} \bigcap_{\mu(N)=0} \operatorname{co} f(t,\{x\} \dot{+} B(\delta) \backslash N),
$$

where the intersections are taken over all sets $N \subset \mathbb{R}^{n}$ of measure zero $(\mu(N)=$ $0)$ and all $\delta>0, \operatorname{co}(M)$ denotes the convex closure of the set $M$.

\subsubsection{Utkin definition (equivalent control method)}

The modification of Filippov definition, which delivers an important impact to the sliding mode control theory, is called the equivalent control method [3].

Consider the system

$$
\dot{x}=f(t, x, u(t, x)), \quad t \in \mathbb{R},
$$

where $f: \mathbb{R} \times \mathbb{R}^{n} \times \mathbb{R}^{m} \rightarrow \mathbb{R}^{n}$ is a continuous vector-valued function and a piecewise continuous function

$$
u: \mathbb{R} \times \mathbb{R}^{n} \rightarrow \mathbb{R}^{m}, \quad u(t, x)=\left(u_{1}(t, x), u_{2}(t, x), \ldots, u_{m}(t, x)\right)^{T}
$$

has a sense of a feedback control. 
Assumption 1. Each component $u_{i}(t, x)$ is discontinuous only on a surface

$$
\mathcal{S}_{i}=\left\{(t, x) \in \mathbb{R}^{n}: s_{i}(t, x)=0\right\},
$$

where functions $s_{i}: \mathbb{R}^{n+1} \rightarrow \mathbb{R}$ are smooth, i.e. $s_{i} \in \mathbb{C}^{1}\left(\mathbb{R}^{n+1}\right)$.

Introduce the following differential inclusion

$$
\dot{x} \in f(t, x, K[u](t, x)), \quad t \in \mathbb{R},
$$

where

$$
K\left[u_{i}\right](t, x)=\left\{\begin{array}{cc}
K[u](t, x)=\left(K\left[u_{1}\right](t, x), \ldots, K\left[u_{m}\right](t, x)\right)^{T}, & s_{i}(t, x) \neq 0, \\
\operatorname{co}\left\{u_{i}(t, x)\right\}, & s_{i}(t, x)=0 . \\
\left.\lim _{\substack{\left(t_{j}, x_{j}\right) \rightarrow(t, x) \\
s_{i}\left(t_{j}, x_{j}\right)>0}} u_{i}\left(t_{j}, x_{j}\right), \lim _{\substack{\left(t_{j}, x_{j}\right) \rightarrow(t, x) \\
s_{i}\left(t_{j}, x_{j}\right)<0}} u_{i}\left(t_{j}, x_{j}\right)\right\}
\end{array}\right.
$$

The set $f\left(t, x, K\left[u_{1}\right](t, x), \ldots, K\left[u_{m}\right](t, x)\right)$ is non-convex in general case [11].

Definition 2. An absolutely continuous function $x: \mathcal{I} \rightarrow \mathbb{R}^{n}$ defined on some interval or segment $\mathcal{I}$ is called a solution of (13) if there exists a measurable function $u_{e q}: \mathcal{I} \rightarrow \mathbb{R}^{m}$ such that $u_{e q}(t) \in K[u](t, x(t))$ and $\dot{x}(t)=$ $f\left(t, x(t), u_{e q}(t)\right)$ almost everywhere on $\mathcal{I}$.

The given definition introduces a solution of the differential equation (13), which we call Utkin solution, since it follows the basic idea of the equivalent control method introduced by V.I. Utkin [3], page 14 (see also [7], page 54).

Obviously, for $(t, x(t)) \notin \mathcal{S}$ we have $u_{e q}(t)=u(t, x(t))$. So, the only question is how to define $u_{e q}(t)$ on a switching surface. The scheme presented in [3] is based on resolving of the equation $\dot{s}(t, x)=\frac{\partial s}{\partial t}+\nabla^{T} s(x) f\left(t, x, u_{e q}\right)=0$ in algebraic way. The obtained solution $u_{e q}(t, x)$ is called equivalent control [3].

In order to show a difference between Utkin and Filippov definitions we consider the system (13) with $u \in \mathbb{R}(m=1)$ and a time-invariant switching surface $\mathcal{S}=\left\{x \in \mathbb{R}^{n}: s(x)=0\right\}$. 
Denote

$$
\begin{gathered}
u^{+}(t, x)=\lim _{x_{j} \rightarrow x, s\left(x_{j}\right)>0} u\left(t, x_{j}\right) \quad \text { and } \quad u^{-}(t, x)=\lim _{x_{j} \rightarrow x, s\left(x_{j}\right)<0} u\left(t, x_{j}\right), \\
f^{+}(t, x)=f\left(t, x, u^{+}(t, x)\right) \quad \text { and } \quad f^{-}(t, x)=f\left(t, x, u^{-}(t, x)\right) .
\end{gathered}
$$

The sliding mode existence condition

$$
\exists \mu \in[0,1]: \nabla s(x) \perp \mu f^{-}(t, x)+(1-\mu) f^{+}(t, x)
$$

is the same for both definitions.

The sliding motion equation obtained by Filippov definition has the form (9) recalled here by

$$
\begin{gathered}
\dot{x}=f_{0}(t, x), \\
f_{0}(t, x)=\frac{\left\langle\nabla s(x), f^{-}(t, x)\right\rangle f^{+}(t, x)+\left\langle\nabla s(x), f^{+}(t, x)\right\rangle f^{-}(t, x)}{\left\langle\nabla s(x), f^{+}(t, x)-f^{-}(t, x)\right\rangle} .
\end{gathered}
$$

The corresponding vector $f_{0}(t, x)$ is defined by a cross-point of the tangential plane at the point $x \in \mathcal{S}$ and a segment connecting the ends of the vectors $f^{+}(t, x)$ and $f^{-}(t, x)$ (see Fig. 3(a)).

Utkin definition considers a set $K[u](t, x)$, which is the convex closure of a set of limit values of a discontinuous control function $u(t, x)$. For different $u_{1}, u_{2}, u_{3}, \ldots \in K[u](t, x)$ the vectors $f\left(t, x, u_{1}\right), f\left(t, x, u_{2}\right), f\left(t, x, u_{3}\right), \ldots$ end on an arc connecting the ends of the vectors $f^{+}(t, x)$ and $f^{-}(t, x)$ (see Fig. 3(b)). In this case the vector $f\left(t, x, u_{e q}\right)$ defining the right-hand side of the sliding motion equation is derived by a cross-point of this arc and a tangential plane at the point $x \in \mathcal{S}$ (see Fig. 3(b)), i.e.

$$
\dot{x}=f\left(t, x, u_{e q}(t, x)\right), \quad x \in \mathcal{S},
$$

where $u_{e q}(t, x) \in K[u](t, x): \nabla s(x) \perp f\left(t, x, u_{e q}(t, x)\right)$.

Sometimes Utkin definition gives quite strange, from mathematical point of view, results, but they are very consistent with real-life applications.

Example 2. ([7]) Consider the system

$$
\dot{x}=A x+b u_{1}+c u_{2}, \quad u_{1}=\operatorname{sign}\left[x_{1}\right], \quad u_{2}=\operatorname{sign}\left[x_{1}\right],
$$


where $x=\left(x_{1}, x_{2}, \ldots, x_{n}\right)^{T} \in \mathbb{R}^{n}, A \in \mathbb{R}^{n \times n}, c, b \in \mathbb{R}^{n}, c \neq b$. Filippov definition provides the inclusion

$$
\dot{x} \in\{A x\} \dot{+}(b+c) \cdot \overline{\operatorname{sign}}\left[x_{1}\right],
$$

where $\dot{+}$ is the geometric (Minkovski) sum of sets (see (3)), $\overline{\operatorname{sign}}$ is the set-valued modification of the sign function (see (2)) and the product of a vector to a set is defined by (5).

If the functions $u_{1}$ and $u_{2}$ are independent control inputs, then Utkin definition gives

$$
\dot{x} \in\{A x\} \dot{+} b \cdot \overline{\operatorname{sign}}\left[x_{1}\right] \dot{+} c \cdot \overline{\operatorname{sign}}\left[x_{1}\right] .
$$

The right-hand sides of (18) and (19) coincide if the vectors $c$ and $b$ are collinear, otherwise Filippov and Utkin definitions generate different set-valued mappings.

For example, if $x=\left(x_{1}, x_{2}\right)^{T} \in \mathbb{R}^{2}, A=0, b=(-1,0)^{T}$ and $c=(0,-1)^{T}$, then

a) Filippov definition gives $K[f](x)=[-1,1] \cdot\left(\begin{array}{l}1 \\ 1\end{array}\right)$ for $x_{1}=0$, i.e. $K[f](x)$ is a segment connecting the points $(-1,-1)$ and $(1,1)$ (see Fig. 2(a)); the corresponding sliding motion equation is

$$
\dot{x}=0 \quad \text { for } \quad x_{1}=0 ;
$$

b)Utkin definition generates the square box, i.e. $K[f](x)=[-1,1] \times[-1,1]$ for $x_{1}=0$ (see Fig. 2(b)), so sliding motion equation has the form

$$
\dot{x}=\left(\begin{array}{c}
0 \\
u_{e q}(t)
\end{array}\right) \quad \text { for } \quad x_{1}=0
$$

where $u_{e q}: \mathbb{R} \rightarrow \mathbb{R}$ is an arbitrary locally measurable function such that $\left|u_{e q}(t)\right| \leq$ 1 for every $t \in \mathbb{R}$.

Control inputs $u_{1}$ and $u_{2}$ are independent and relay elements are not identical in practice. They can not switch absolutely synchronously. This admits a motion of the system along the switching line $x_{1}=0$. In this case, Utkin definition is more adequate to reality than Filippov one. 


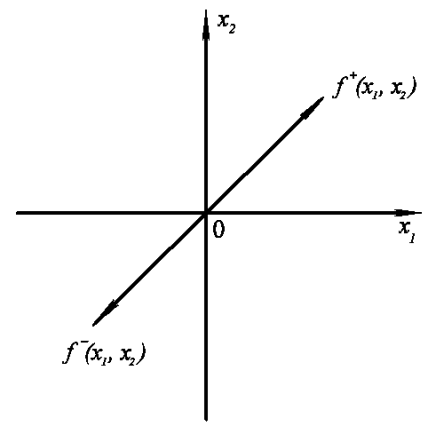

(a) Filippov definition.

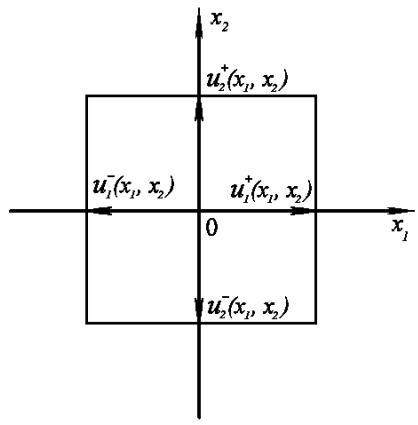

(b) Utkin definition.

Figure 2: Example of Filippov's and Utkin's sets.

\subsubsection{Aizerman-Pyatnitskii definition}

The Aizerman-Pyatnitskii definition covers solutions of both definitions considered above by means of introduction of the following differential inclusion

$$
\dot{x} \in \operatorname{co} f(t, x, K[u](t, x)), \quad t \in \mathbb{R}
$$

for the system (13).

Definition 3 (Aizerman-Pyatnitskii definition ([10] and [7], page 55)). An absolutely continuous function $x: \mathcal{I} \rightarrow \mathbb{R}^{n}$ defined on some interval or segment $\mathcal{I}$ is called a solution of (6) if it satisfies the differential inclusion (20) almost everywhere on $\mathcal{I}$.

Returning to the example considered above for $u \in \mathbb{R}(m=1)$ AizermanPyatnitskii definition gives the inclusion

$$
\dot{x} \in F_{S M}(t, x)=\operatorname{co}\left\{f_{0}(t, x), f\left(t, x, u_{e q}(t, x)\right)\right\},
$$

which describes the motion of the discontinuous system (13) in a sliding mode (see Fig. 3(c) with $f_{\alpha} \in F_{S M}(t, x)$ ).

A criticism of Aizerman-Pyatnitskii definition is related to nonuniqueness of solutions even for simple nonlinear cases. However, if some stability property is proven for Aizerman-Pyatnickii definition, then the same property holds for both Filippov and Utkin solutions. 


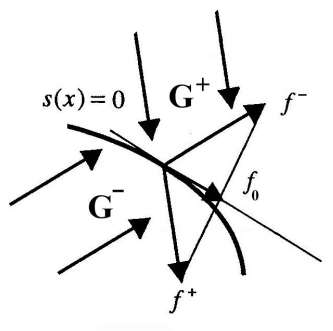

(a) Filippov definition.

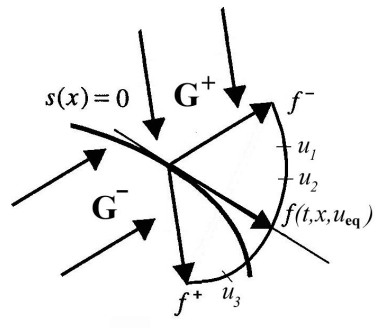

(b) Utkin definition.

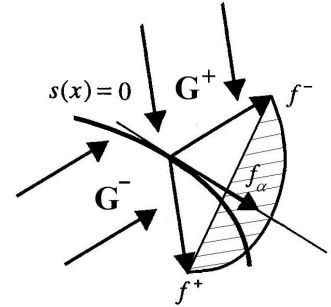

(c) Aizerman-Pyatnitskii definition.

Figure 3: The sliding motion for different definitions.

The affine control system is the case when all definitions may be equivalent.

Theorem 1 ([37], Theorem 14, page 44). Let a right-hand side of the system (6) be affine with respect to control:

$$
f(t, x)=a(t, x)+b(t, x) u(t, x),
$$

where $a: \mathbb{R}^{n+1} \rightarrow \mathbb{R}^{n}$ is a continuous vector-valued function, $b: \mathbb{R}^{n+1} \rightarrow \mathbb{R}^{n \times m}$ is a continuous matrix-valued function and $u: \mathbb{R}^{n+1} \rightarrow \mathbb{R}^{m}$ is a piecewise continuous function $u(t, x)=\left(u_{1}(t, x), \ldots, u_{m}(t, x)\right)^{T}$, such that $u_{i}$ has a unique time-invariant switching surface $s_{i}(x)=0, s_{i} \in \mathbb{C}^{1}\left(\mathbb{R}^{n}\right)$.

Definitions of Filippov, Utkin and Aizerman-Pyatnitskii are equivalent iff

$$
\operatorname{det}\left(\nabla^{T} s(x) b(t, x)\right) \neq 0 \quad \text { if } \quad(t, x) \in \mathcal{S},
$$

where $s(x)=\left(s_{1}(x), s_{2}(x), \ldots, s_{m}(x)\right)^{T}, \nabla s(x) \in \mathbb{R}^{n \times m}$ is the matrix of partial derivatives $\frac{\partial s_{j}}{\partial x_{i}}$ and $\mathcal{S}$ is a discontinuity set of $u(t, x)$.

The present theorem has the simple geometric interpretation for the single input system. The affine control system is linear with respect to the control input, which is the only discontinuous term of the right-hand side of the system (6). In this case all regularization procedures provide the set-valued extension depicted on Fig. 3(a). The condition (21) excludes non-uniqueness of this setvalued extension for multi-input case. For example, the system considered in Example 17 is affine, but it does not satisfy the condition (21). 


\subsection{System disturbances and extended differential inclusion}

Some modifications of presented definitions of solutions are required again if a model of a dynamic system includes disturbances into considerations. For example, the paper [12] extends Filippov definition to discontinuous disturbed systems. It demonstrates that the presented extension is useful for ISS analysis.

The present survey is mostly oriented on sliding mode control systems. The robustness of sliding mode control systems (at least theoretically) is related to invariance of qualitative behavior of closed-loop system on matched disturbances with some a priori known maximum magnitude [3], [8], [6]. This property usually allows reducing a problem of stability analysis of a disturbed discontinuous sliding mode control system to a similar problem presented for an extended differential inclusion. The idea explained in the next example was also used in papers [15], [38].

Example 3. Consider the simplest disturbed sliding mode system

$$
\dot{x}=-d_{1}(t) \operatorname{sign}[x]+d_{2}(t),
$$

where $x \in \mathbb{R}$, unknown functions $d_{i}: \mathbb{R} \rightarrow \mathbb{R}$ are bounded by

$$
d_{i}^{\min } \leq d_{i}(t) \leq d_{i}^{\max }, \quad i=1,2,
$$

and the function $\operatorname{sign}[x]$ is defined by (1).

Obviously, all solutions of the system (22) belong to a solution set of the following extended differential inclusion

$$
\dot{x} \in-\left[d_{1}^{\min }, d_{1}^{\max }\right] \cdot \overline{\operatorname{sign}}[x]+\left[d_{2}^{\min }, d_{2}^{\max }\right] .
$$

Stability of the system (24) implies the same property for (22). In particular, for $d_{1}^{\min }>\max \left\{\left|d_{2}^{\min }\right|,\left|d_{2}^{\max }\right|\right\}$ both these systems have asymptotically stable origins.

This example shows that the conventional properties, like asymptotic or finite stability, discovered for differential inclusions may provide "robust" stability 
for original discontinuous differential equations. That is why, in this paper we $d o$ not discuss "robust" modifications of stability notions for differential inclusions.

Models of sliding mode control systems usually have the form

$$
\dot{x}=f(t, x, u(t, x), d(t)), \quad t \in \mathbb{R},
$$

where $x \in \mathbb{R}^{n}$ is the vector of system states, $u \in \mathbb{R}^{m}$ is the vector of control inputs, $d \in \mathbb{R}^{k}$ is the vector of disturbances, the function $f: \mathbb{R}^{n+m+k+1} \rightarrow \mathbb{R}^{n}$ is assumed to be continuous, the control function $u: \mathbb{R}^{n+1} \rightarrow \mathbb{R}^{m}$ is piecewise continuous, the vector-valued function $d: \mathbb{R} \rightarrow \mathbb{R}^{k}$ is assumed to be locally measurable and bounded as follows:

$$
d_{i}^{\min } \leq d_{i}(t) \leq d_{i}^{\max },
$$

where $d(t)=\left(d_{1}(t), d_{2}(t), \ldots, d_{k}(t)\right)^{T}, t \in \mathbb{R}$.

All further considerations deal with the extended differential inclusion

$$
\dot{x} \in F(t, x), \quad t \in \mathbb{R},
$$

where $F(t, x)=\operatorname{co}\{f(t, x, K[u](t, x), D)\}$, the set-valued function $K[u](t, x)$ is defined by (15) and

$$
D=\left\{\left(d_{1}, d_{2}, \ldots, d_{k}\right)^{T} \in \mathbb{R}^{k}: d_{i} \in\left[d_{i}^{\min }, d_{i}^{\max }\right], i=1,2, \ldots, k\right\} .
$$

The same extended differential inclusion can be used if the vector $d$ (or its part) has a sense of parametric uncertainties.

\subsection{Existence of solutions}

Let us recall initially the classical result of Caratheodory about existence of solutions for ODEs with right-hand sides, which are discontinuous on time.

Theorem 2 ([33], Theorem 1.1, Chapter 2). Let the function

$$
\begin{gathered}
g: \mathbb{R} \times \mathbb{R}^{n} \rightarrow \mathbb{R}^{n} \\
(t, x) \rightarrow g(t, x)
\end{gathered}
$$

be continuous by $x$ in $\Omega=\left\{x_{0}\right\}+\mathcal{B}(r), r \in \mathbb{R}_{+}, x_{0} \in \mathbb{R}^{n}$ for any fixed $t \in \mathcal{I}=$ $\left[t_{0}-a, t_{0}+a\right], a \in \mathbb{R}_{+}, t_{0} \in \mathbb{R}$ and it is measurable by $t$ for any fixed $x \in \Omega$. If 
there exists an integrable function $m: \mathbb{R} \rightarrow \mathbb{R}$ such that $\|f(t, x)\| \leq m(t)$ for all $(t, x) \in \mathcal{I} \times \Omega$ then there exists an absolutely continuous function $x: \mathbb{R} \rightarrow \mathbb{R}^{n}$ and $a$ number $b \in(0, a]$ such that $x\left(t_{0}\right)=x_{0}$ and the equality

$$
\dot{x}(t)=g(t, x(t))
$$

hold almost everywhere on $\left[t_{0}-b, t_{0}+b\right]$.

Introduce the following distances

$$
\begin{gathered}
\rho(x, \mathbf{M})=\inf _{y \in \mathbf{M}}\|x-y\|, \quad x \in \mathbb{R}^{n}, \quad \mathbf{M} \subseteq \mathbb{R}^{n}, \\
\rho\left(\mathbf{M}_{1}, \mathbf{M}_{2}\right)=\sup _{x \in \mathbf{M}_{1}} \rho\left(x, \mathbf{M}_{2}\right), \quad \mathbf{M}_{1} \subseteq \mathbb{R}^{n}, \quad \mathbf{M}_{2} \subseteq \mathbb{R}^{n} .
\end{gathered}
$$

Remark, the distance $\rho\left(\mathbf{M}_{1}, \mathbf{M}_{2}\right)$ is not symmetric, i.e. $\rho\left(\mathbf{M}_{1}, \mathbf{M}_{2}\right) \neq \rho\left(\mathbf{M}_{2}, \mathbf{M}_{1}\right)$ in the general case.

Definition 4. A set-valued function $F: \mathbb{R}^{n+1} \rightarrow 2^{\mathbb{R}^{n+1}}$ is said to be upper semi-continuous at a point $\left(t^{*}, x^{*}\right) \in \mathbb{R}^{n+1}$ if $(t, x) \rightarrow\left(t^{*}, x^{*}\right)$ implies

$$
\rho\left(F(t, x), F\left(t^{*}, x^{*}\right)\right) \rightarrow 0
$$

For instance, the function $\overline{\operatorname{sign}}[x]$ defined by (2) is upper semi-continuous.

Theorem 3 ([7], page 77). Let a set-valued function $F: G \rightarrow 2^{\mathbb{R}^{n}}$ be defined and upper semi-continuous at each point of the set

$$
\boldsymbol{G}=\left\{(t, x) \in \mathbb{R}^{n+1}:\left|t-t_{0}\right| \leq a \text { and }\left\|x-x_{0}\right\| \leq b\right\},
$$

where $a, b \in \mathbb{R}_{+}, t_{0} \in \mathbb{R}, x_{0} \in \mathbb{R}^{n}$. Let $F(t, x)$ be nonempty, compact and convex for $(t, x) \in \boldsymbol{G}$.

If there exists $K>0$ such that $\rho(0, F(t, x))<K$ for $(t, x) \in \boldsymbol{G}$ then there exists at least one absolutely continuous function $x: \mathbb{R} \rightarrow \mathbb{R}^{n}$ defined at least on the segment $\left[t_{0}-\alpha, t_{0}+\alpha\right], \alpha=\min \{a, b / K\}$, such that $x\left(t_{0}\right)=x_{0}$ and the inclusion $\dot{x}(t) \in F(t, x(t))$ holds almost everywhere on $\left[t_{0}-\alpha, t_{0}+\alpha\right]$.

Filippov and Aizerman-Pyatnickii set-valued extensions of the discontinuous ODE (see formulas (7) and (20)) and the extended differential inclusion (27) 
satisfy all conditions of Theorem 3 implying local existence of the corresponding solutions.

The existence analysis of Utkin solutions is more complicated in general case. Since the function $f(t, x, u)$ is continuous, then for any measurable bounded function $u_{0}: \mathcal{I} \rightarrow \mathbb{R}^{m}$ the composition $f\left(t, x, u_{0}(t)\right)$ satisfies all conditions of Theorem 2 and the equation $\dot{x}=f\left(t, x, u_{0}(t)\right)$ has an absolutely continuous solution $x_{0}(t)$, but $u_{0}(t)$ may not belong to the set $K[u]\left(t, x_{0}(t)\right)$.

In some cases, the existence of Utkin solution can be proven using the celebrated Filippov's lemma.

Lemma 1 ([39], page 78). Let a function $f: \mathbb{R}^{n+m+1} \rightarrow \mathbb{R}^{n}$ be continuous and a set-valued function $U: \mathbb{R}^{n+1} \rightarrow 2^{\mathbb{R}^{m}}$ be defined and upper-semicontinuous on an open set $\mathcal{I} \times \Omega$, where $\Omega \subseteq \mathbb{R}^{n}$. Let $U(t, x)$ be nonempty, compact and convex for every $(t, x) \in \mathcal{I} \times \Omega$. Let a function $x: \mathbb{R} \rightarrow \mathbb{R}^{n}$ be absolutely continuous on $\mathcal{I}, x(t) \in \Omega$ for $t \in \mathcal{I}$ and $\dot{x}(t) \in f(t, x(t), U(t, x(t)))$ almost everywhere on $\mathcal{I}$.

Then there exists a measurable function $u_{e q}: \mathbb{R} \rightarrow \mathbb{R}^{m}$ such that $u_{e q}(t) \in$ $U(t, x(t))$ and $\dot{x}(t)=f\left(t, x(t), u_{e q}(t)\right)$ almost everywhere on $\mathcal{I}$.

If the differential inclusion (14) has a convex right-hand side then Theorem 3 together with Lemma 1 results local existence of Utkin solutions. If the setvalued function $f(t, x, K[u](t, x))$ is non-convex, the existence analysis of Utkin solutions becomes very difficult (see [11] for the details).

Some additional restrictions to right-hand sides are required for a prolongation of solutions. In particular, the famous Winter's theorem (see, for example, [40], page 515) about a non-local existence of solutions of ODE can be expanded to differential inclusions.

Theorem 4 ([41], page 169). Let a set-valued function $F: \mathbb{R}^{n+1} \rightarrow \mathbb{R}^{n+1}$ be defined and upper-semicontinuous in $\mathbb{R}^{n+1}$. Let $F(t, x)$ be nonempty, compact and convex for any $(t, x) \in \mathbb{R}^{n+1}$. 
If there exists a real valued function $L: \mathbb{R}^{+} \cup\{0\} \rightarrow \mathbb{R}^{+} \cup\{0\}$ such that

$$
\rho(0, F(t, x)) \leq L(\|x\|) \quad \text { and } \quad \int_{0}^{+\infty} \frac{1}{L(r)} d r=+\infty,
$$

then for any $\left(t_{0}, x_{0}\right) \in \mathbb{R}^{n+1}$ the system (27) has a solution $x(t): x\left(t_{0}\right)=x_{0}$ defined for all $t \in \mathbb{R}$.

Based on Lyapunov function method, the less conservative conditions for prolongation of solutions are given below.

\section{Stability and convergence rate}

Consider the differential inclusion (27) for $t>t_{0}$ with an initial condition

$$
x\left(t_{0}\right)=x_{0},
$$

where $x_{0} \in \mathbb{R}^{n}$ is given.

Cauchy problem (27), (31) obviously may not have a unique solution for a given $t_{0} \in \mathbb{R}$ and a given $x_{0} \in \mathbb{R}^{n}$. Let us denote the set of all solutions of Cauchy problem (27), (31) by $\Phi\left(t_{0}, x_{0}\right)$ and a solution of $(27)$, (31) by $x\left(t, t_{0}, x_{0}\right) \in$ $\Phi\left(t_{0}, x_{0}\right)$.

Nonuniqueness of solutions implies two types of stability for differential inclusions (27): weak stability(a property holds for a solution) and strong stability (a property holds for all solutions) (see, for example, [27], [13], [7]). Weak stability usually is not enough for robust control purposes. This section observes only strong stability properties of the system (27). All conditions presented in definitions below are assumed to be held for all solutions $x\left(t, t_{0}, x_{0}\right) \in \Phi\left(t_{0}, x_{0}\right)$.

\subsection{Lyapunov, asymptotic and exponential stability}

The concept of stability introduced in the famous thesis of A.M. Lyapunov [17] is one of central notions of the modern stability theory. It considers some nominal motion $x^{*}\left(t, t_{0}, x_{0}\right)$ of a dynamic system and studies small perturbations of the initial condition $x_{0}$. If they imply small deviations of perturbed motions from $x^{*}\left(t, t_{0}, x_{0}\right)$ then the nominal motion is called stable. We study different stability forms of the zero solution (or, equivalently, the origin) of the 
system (27), since making the change of variables $y=x-x^{*}$ we transform any problem of stability analysis for some nontrivial solution $x^{*}\left(t, t^{*}, x_{0}^{*}\right)$ to the same problem for the zero solution.

Assume that $0 \in F(t, 0)$ for $t \in \mathbb{R}$, where $F(t, x)$ is defined by $(27)$. Then the function $x_{0}(t)=0$ belongs to a solution set $\Phi\left(t, t_{0}, 0\right)$ for any $t_{0} \in \mathbb{R}$.

Definition 5 (Lyapunov stability). The origin of the system (27) is said to be Lyapunov stable if for $\forall \varepsilon \in \mathbb{R}_{+}$and $\forall t_{0} \in \mathbb{R}$ there exists $\delta=\delta\left(\varepsilon, t_{0}\right) \in \mathbb{R}_{+}$ such that for $\forall x_{0} \in \mathcal{B}(\delta)$

1) any solution $x\left(t, t_{0}, x_{0}\right)$ of Cauchy problem (27), (31) exists for $t>t_{0}$;

2) $x\left(t, t_{0}, x_{0}\right) \in \mathcal{B}(\varepsilon)$ for $t>t_{0}$.

If the function $\delta$ does not depend on $t_{0}$ then the origin is called uniformly Lyapunov stable. For instance, if $F(t, x)$ is independent of $t$ (time-invariant case) and the zero solution of (27) is Lyapunov stable, then it is uniformly Lyapunov stable.

Proposition 1. If the origin of the system (27) is Lyapunov stable then $x(t)=$ 0 is the unique solution of Cauchy problem (27), (31) with $x_{0}=0$ and $t_{0} \in \mathbb{R}$.

The origin, which does not satisfy any condition from Definition 5, is called unstable.

Definition 6 (Asymptotic attractivity). The origin of the system (27) is said to be asymptotically attractive if for $\forall t_{0} \in \mathbb{R}$ there exists a set $\mathcal{U}\left(t_{0}\right) \subseteq \mathbb{R}^{n}$ : $0 \in \operatorname{int}\left(\mathcal{U}\left(t_{0}\right)\right)$ such that $\forall x_{0} \in \mathcal{U}\left(t_{0}\right)$

- any solution $x\left(t, t_{0}, x_{0}\right)$ of Cauchy problem (27), (31) exists for $t>t_{0}$;

- $\lim _{t \rightarrow+\infty}\left\|x\left(t, t_{0}, x_{0}\right)\right\|=0$.

The set $\mathcal{U}\left(t_{0}\right)$ is called attraction domain.

Finding the maximum attraction domain is an important problem for many practical control applications. 
Definition 7 (Asymptotic stability). The origin of the system (27) is said to be asymptotically stable if it is Lyapunov stable and asymptotically attractive.

If $\mathcal{U}\left(t_{0}\right)=\mathbb{R}^{n}$ then the asymptotically stable (attractive) origin of the system (27) is called globally asymptotically stable (attractive).

Requirement of Lyapunov stability is very important in Definition 7 , since even global asymptotic attractivity does not imply Lyapunov stability.

Example 4 ([42], page 433 or [43], page 191). The system

$$
\dot{x}_{1}=\frac{x_{1}^{2}\left(x_{2}-x_{1}\right)+x_{2}^{5}}{\left(x_{1}^{2}+x_{2}^{2}\right)\left(1+\left(x_{1}^{2}+x_{2}^{2}\right)^{2}\right)} \quad \text { and } \quad \dot{x}_{2}=\frac{x_{2}^{2}\left(x_{2}-2 x_{1}\right)}{\left(x_{1}^{2}+x_{2}^{2}\right)\left(1+\left(x_{1}^{2}+x_{2}^{2}\right)^{2}\right)}
$$

has the globally asymptotically attractive origin. However, it is not Lyapunov stable, since this system has trajectories (see Fig. 4), which start in arbitrary small ball with the center at the origin and always leave the ball $\mathcal{B}\left(\varepsilon_{0}\right)$ of a fixed radius $\varepsilon_{0} \in \mathbb{R}_{+}$(i.e. Condition 2 of Definition 5 does not hold for $\varepsilon \in\left(0, \varepsilon_{0}\right)$ ).

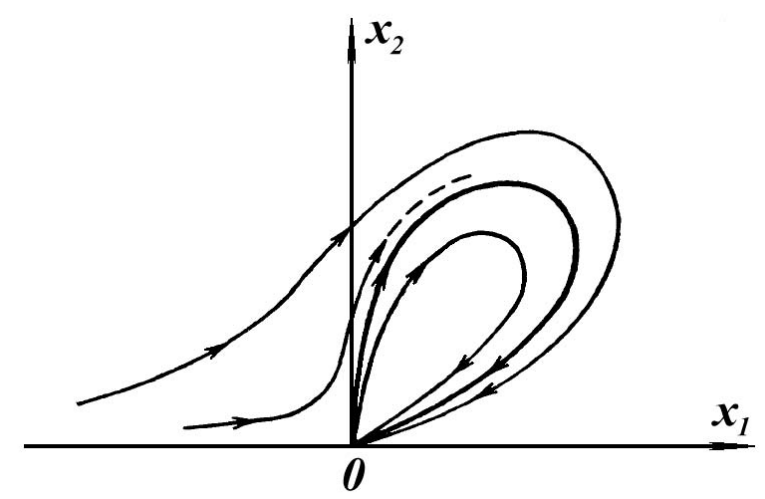

Figure 4: Example of R.E. Vinograd [42].

The uniform asymptotic stability can be introduced by analogy with uniform Lyapunov stability. It just requests more strong attractivity property.

Definition 8 (Uniform asymptotic attractivity). The origin of the system (27) is said to be uniformly asymptotically attractive if it is asymptotically attractive with a time-invariant attraction domain $\mathcal{U} \subseteq \mathbb{R}^{n}$ and for $\forall R \in \mathbb{R}_{+}$, 
$\forall \varepsilon \in \mathbb{R}_{+}$there exists $T=T(R, \varepsilon) \in \mathbb{R}_{+}$such that the inclusions $x_{0} \in \mathcal{B}(R) \cap \mathcal{U}$ and $t_{0} \in \mathbb{R}$ imply $x\left(t, t_{0}, x_{0}\right) \in \mathcal{B}(\varepsilon)$ for $t>t_{0}+T$.

Definition 9 (Uniform asymptotic stability). The origin of the system (27) is said to be uniformly asymptotically stable if it is uniformly Lyapunov stable and uniformly asymptotically attractive.

If $\mathcal{U}=\mathbb{R}^{n}$ then a uniformly asymptotically stable (attractive) origin of the system (27) is called globally uniformly asymptotically stable (attractive). Uniform asymptotic stability always implies asymptotic stability. The converse proposition also holds for time-invariant systems.

Proposition 2 ([44], Proposition 2.2, page 78). Let a set-valued function $F: R^{n} \rightarrow \mathbb{R}^{n}$ be defined and upper-semicontinuous in $\mathbb{R}^{n}$. Let $F(x)$ be nonempty, compact and convex for any $x \in \mathbb{R}^{n}$. If the origin of the system

$$
\dot{x} \in F(x)
$$

is asymptotically stable then it is uniformly asymptotically stable.

Frequently, an asymptotic stability of a closed-loop system is not enough for a "good" quality of control. A rate of transition processes also has to be adjusted in order to provide a better performance to a control system. For this purpose some concepts of "rated" stability can be used such as exponential, finite-time or fixed-time stability.

Definition 10 (Exponential stability). The origin of the system (27) is said to be exponentially stable if there exist an attraction domain $\mathcal{U} \subseteq \mathbb{R}^{n}: 0 \in \operatorname{int}(\mathcal{U})$ and numbers $C, r \in \mathbb{R}_{+}$such that

$$
\left\|x\left(t, t_{0}, x_{0}\right)\right\| \leq C\left\|x_{0}\right\| e^{-r\left(t-t_{0}\right)}, t>t_{0} .
$$

for $t_{0} \in \mathbb{R}$ and $x_{0} \in \mathcal{U}$.

The inequality (32) expresses the so-called exponential convergence (attractivity) property. The linear control theory usually deals with this property [19].

Exponential stability obviously implies both Lyapunov stability and asymptotic stability. 


\subsection{Finite-time Stability}

Introduce the functional $T_{0}: \mathbb{W}_{\left[t_{0},+\infty\right)}^{n} \rightarrow \overline{\mathbb{R}}_{+} \cup\{0\}$ by the following formula

$$
T_{0}(y(\cdot))=\inf _{\tau \geq t_{0}: y(\tau)=0} \tau .
$$

If $y(\tau) \neq 0$ for all $t \in\left[t_{0},+\infty\right)$ then $T_{0}(y(\cdot))=+\infty$.

Let us define the settling-time function of the system (27) as follows

$$
T\left(t_{0}, x_{0}\right)=\sup _{x\left(t, t_{0}, x_{0}\right) \in \Phi\left(t_{0}, x_{0}\right)} T_{0}\left(x\left(t, t_{0}, x_{0}\right)\right)-t_{0},
$$

where $\Phi\left(t_{0}, x_{0}\right)$ is the set of all solutions of the Cauchy problem (27), (31).

Definition 11 (Finite-time attractivity). The origin of the system (27) is said to be finite-time attractive if for $\forall t_{0} \in \mathbb{R}$ there exists a set $\mathcal{V}\left(t_{0}\right) \subseteq \mathbb{R}^{n}: 0 \in$ $\operatorname{int}\left(\mathcal{V}\left(t_{0}\right)\right)$ such that $\forall x_{0} \in \mathcal{V}\left(t_{0}\right)$

- any solution $x\left(t, t_{0}, x_{0}\right)$ of Cauchy problem (27), (31) exists for $t>t_{0}$;

- $T\left(t_{0}, x_{0}\right)<+\infty$ for $x_{0} \in \mathcal{V}\left(t_{0}\right)$ and for $t_{0} \in R$.

The set $\mathcal{V}\left(t_{0}\right)$ is called finite-time attraction domain.

It is worth to stress that the finite-time attractivity property, introduced originally in [14], does not imply asymptotic attractivity. However, it is important for many control applications. For example, antimissile control problem has to be studied only on a finite interval of time, since there is nothing to control after missile explosion. In practice, Lyapunov stability is additionally required in order to guarantee a robustness of a control system.

Definition 12 (Finite-time stability ([13], [14])). The origin of the system (27) is said to be finite-time stable if it is Lyapunov stable and finite-time attractive.

If $\mathcal{V}\left(t_{0}\right)=\mathbb{R}^{n}$ then the origin of (27) is called globally finite-time stable. 
Example 5. Consider the sliding mode system

$$
\dot{x}=-\frac{2}{\sqrt{\pi}} \operatorname{sign}[x]+|2 t x|, \quad t>t_{0}, \quad x \in \mathbb{R},
$$

which, according to Filippov definition, is extended to the differential inclusion

$$
\dot{x} \in-\frac{2}{\sqrt{\pi}} \cdot \overline{\operatorname{sign}}[x] \dot{+}\{|2 t x|\}, \quad t>t_{0}, \quad x \in \mathbb{R},
$$

where $t_{0} \in \mathbb{R}$. It can be shown that the origin of this system is finite-time attractive with an attraction domain $\mathcal{V}\left(t_{0}\right)=\mathcal{B}\left(e^{t_{0}^{2}}\left(1-\operatorname{erf}\left(\left|t_{0}\right|\right)\right)\right)$, where

$$
\operatorname{erf}(z)=\frac{2}{\sqrt{\pi}} \int_{0}^{z} e^{-\tau^{2}} d \tau, \quad z \in \mathbb{R}
$$

is the so-called Gauss error function. Moreover, the origin of the considered system is Lyapunov stable (for $\forall \varepsilon>0$ and for $\forall t_{0} \in \mathbb{R}$ we can select $\delta=$ $\left.\delta\left(t_{0}\right)=\min \left\{\varepsilon, e^{t_{0}^{2}}\left(1-\operatorname{erf}\left(\left|t_{0}\right|\right)\right)\right\}\right)$, so it is finite-time stable. In particular, for $t_{0}>0$ the settling-time function has the form

$$
T\left(t_{0}, x_{0}\right)=\operatorname{erf}^{-1}\left(\left|x_{0}\right| e^{-t_{0}^{2}}+\operatorname{erf}\left(t_{0}\right)\right)-t_{0},
$$

where $\operatorname{erf}^{-1}(\cdot)$ denotes the inverse function to $\operatorname{erf}(\cdot)$.

The proposition 1 implies the following property of a finite-time stable system.

Proposition 3 ([14], Proposition 2.3). If the origin of the system (27) is finite-time stable then it is asymptotically stable and $x\left(t, t_{0}, x_{0}\right)=0$ for $t>$ $t_{0}+T_{0}\left(t_{0}, x_{0}\right)$.

A uniform finite-time attractivity requests an additional property for the system (27).

Definition 13 (Uniform finite-time attractivity). The origin of the system (27) is said to be uniformly finite-time attractive if it is finite-time attractive with a time-invariant attraction domain $\mathcal{V} \subseteq \mathbb{R}^{n}$ such that the settling time function $T\left(t_{0}, x_{0}\right)$ is locally bounded on $\mathbb{R} \times \mathcal{V}$ uniformly on $t_{0} \in$ $\mathbb{R}$, i.e. for any $y \in \mathcal{V}$ there exists $\varepsilon \in \mathbb{R}_{+}$such that $\{y\} \dot{+} \mathcal{B}(\varepsilon) \subseteq \mathcal{V}$ and $\sup _{t_{0} \in \mathbb{R}, x_{0} \in\{y\}+\mathcal{B}(\varepsilon)} T\left(t_{0}, x_{0}\right)<+\infty$. 
Definition 14 (Uniform finite-time stability, [13], [15]). The origin of the system (27) is said to be uniformly finite-time stable if it is uniformly Lyapunov stable and uniformly finite-time attractive.

The origin of (27) is called globally uniformly finite-time stable if $\mathcal{V}=\mathbb{R}^{n}$.

Obviously, a settling-time function of time-invariant finite-time stable system (27) is independent of $t_{0}$, i.e. $T=T\left(x_{0}\right)$. However, in contrast to asymptotic and Lyapunov stability, finite-time stability of a time-invariant system does not imply its uniform finite-time stability in general case.

Example 6 ([14], page 756). Let a vector field $f: \mathbb{R}^{2} \rightarrow \mathbb{R}^{2}$ of a timeinvariant system be defined on the quadrants

$$
\begin{array}{cc}
\mathcal{Q}_{I}=\left\{x \in \mathbb{R}^{2} \backslash\{0\}: x_{1} \geq 0, x_{2} \geq 0\right\} & \mathcal{Q}_{I I}=\left\{x \in \mathbb{R}^{2}: x_{1}<0, x_{2} \geq 0\right\} \\
\mathcal{Q}_{I I I}=\left\{x \in \mathbb{R}^{2}: x_{1} \leq 0, x_{2}<0\right\} & \mathcal{Q}_{I V}=\left\{x \in \mathbb{R}^{2}: x_{1}>0, x_{2}<0\right\}
\end{array}
$$

as show in Fig. 5. The vector field $f$ is continuous, $f(0)=0$ and $x=$ $\left(x_{1}, x_{2}\right)^{T}=(r \cos (\theta), r \sin (\theta))^{T}, r>0, \theta \in[0,2 \pi)$. In [14] it was shown that

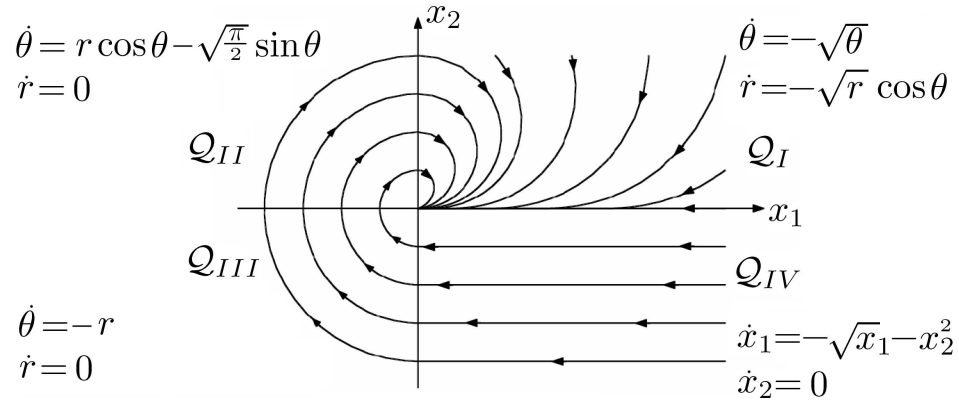

Figure 5: Example of S.P. Bhat and D. Bernstein [14].

this system is finite-time stable. Moreover, it is uniformly asymptotically stable, but it is not uniformly finite-time stable. For the sequence of the initial conditions $x_{0}^{i}=(0,-1 / i)^{T}, i=1,2, \ldots$ we have (see [14] for the details)

$$
x_{0}^{i} \rightarrow 0 \text { and } T\left(x_{0}^{i}\right) \rightarrow+\infty .
$$

So, for any open ball $B(r), r>0$ with the center at the origin we have

$$
\sup _{x_{0} \in B(r)} T\left(x_{0}\right)=+\infty .
$$


Uniform finite-time stability is the usual property for sliding mode systems [15], [38]. The further considerations deals mainly with this property and its modifications.

\subsection{Fixed-time Stability}

This subsection discusses a recent extension of the uniform finite-time stability concept, which is called fixed-time stability [30]. Fixed-time stability asks more strong uniform attractivity property for the system (27). As it was demonstrated in [32], [30], this property is very important for some applications, such as control and observation with predefined convergence time.

In order to demonstrate the necessity of more detailed elaboration of uniformity properties of finite-time stable systems let us consider the following motivating example.

Example 7. Consider two systems

$$
\text { (I) } \dot{x}=-x^{\left[\frac{1}{2}\right]}(1-|x|), \quad(I I) \quad \dot{x}=\left\{\begin{array}{ccc}
-x^{\left[\frac{1}{2}\right]} & \text { for } & x<1, \\
0 & \text { for } & x \geq 1,
\end{array}\right.
$$

which are uniformly finite-time stable with the finite-time attraction domain $\mathcal{V}=\mathcal{B}(1)$. Indeed, the settling-time functions of these systems are continuous on $\mathcal{V}$ :

$$
T_{(I)}\left(x_{0}\right)=\ln \left(\frac{1+\left|x_{0}\right|^{\frac{1}{2}}}{1-\left|x_{0}\right|^{\frac{1}{2}}}\right), \quad T_{(I I)}\left(x_{0}\right)=2\left|x_{0}\right|^{\frac{1}{2}} .
$$

So, for any $y \in \mathcal{V}$ we can select the ball $\{y\}+\mathcal{B}(\varepsilon) \subseteq \mathcal{V}$, where $\varepsilon=(1-|y|) / 2$, such that $\sup _{x_{0} \in\{y\}+\mathcal{B}(\varepsilon)} T_{(I)}\left(x_{0}\right)<+\infty$ and $\sup _{x_{0} \in\{y\}+\mathcal{B}(\varepsilon)} T_{(I I)}\left(x_{0}\right)<+\infty$.

On the other hand, $T_{(I)}\left(x_{0}\right) \rightarrow+\infty$ if $x_{0} \rightarrow \pm 1$, but $T_{(I I)}\left(x_{0}\right) \rightarrow 2$ if $x_{0} \rightarrow \pm 1$. Therefore, these systems have different uniformity properties of finitetime attractivity with respect to domain of initial conditions.

Definition 15 (Fixed-time attractivity). The origin of the system (27) is said to be fixed-time attractive if it is uniformly finite-time attractive with an attraction domain $\mathcal{V}$ and the settling time function $T\left(t_{0}, x_{0}\right)$ is bounded on $\mathbb{R} \times \mathcal{V}$, i.e. there exists a number $T_{\max } \in \mathbb{R}_{+}$such that $T\left(t_{0}, x_{0}\right) \leq T_{\max }$ if $t_{0} \in \mathbb{R}$ and $x_{0} \in \mathcal{V}$. 
Systems (I) and (II) from Example 7 are both fixed-time attractive with respect to attraction domain $\mathcal{B}(r)$ if $r \in(0,1)$, but the system (I) loses this property for the maximum attraction domain $\mathcal{B}(1)$.

Definition 16 (Fixed-time stability, [30]). The origin of the system (27) is said to be fixed-time stable if it is Lyapunov stable and fixed-time attractive.

If $\mathcal{V}=\mathbb{R}^{n}$ then the origin of the system (27) is called globally fixed-time stable. Locally differences between finite-time and fixed-time stability are questionable. Fixed-time stability definitely provides more advantages to a control system in a global case [32], [30].

Example 8. Consider the system

$$
\dot{x}=-x^{\left[\frac{1}{2}\right]}-x^{\left[\frac{3}{2}\right]}, x \in \mathbb{R}, t>t_{0},
$$

which has solutions defined for all $t \geq t_{0}$ :

$x\left(t, t_{0}, x_{0}\right)=\left\{\begin{aligned} \operatorname{sign}\left(x_{0}\right) \tan ^{2}\left(\arctan \left(\left|x_{0}\right|^{\frac{1}{2}}\right)-\frac{t-t_{0}}{2}\right), & t \leq t_{0}+2 \arctan \left(\left|x_{0}\right|^{\frac{1}{2}}\right), \\ 0, & t>t_{0}+2 \arctan \left(\left|x_{0}\right|^{\frac{1}{2}}\right) .\end{aligned}\right.$

Any solution $x\left(t, t_{0}, x_{0}\right)$ of this system converges to the origin in a finite time. Moreover, for any $x_{0} \in \mathbb{R}, t_{0} \in \mathbb{R}$ the equality $x\left(t, t_{0}, x_{0}\right)=0$ holds for all $t \geq t_{0}+\pi$, i.e. the system is globally fixed-time stable with $T_{\max }=\pi$.

\section{Generalized derivatives}

The celebrated Second Lyapunov Method is founded on the so-called energetic approach to stability analysis. It considers any positive definite function as an possible energetic characteristic ("energy") of a dynamic system and studies evolution of this "energy" in time. If a dynamic system has an energetic function, which is decreasing (strongly decreasing or bounded) along any trajectory of the system, then this system has a stability property and the corresponding energetic function is called Lyapunov function.

For example, to analyze asymptotic stability of the origin of the system

$$
\dot{x}=f(t, x), \quad f \in \mathbb{C}\left(\mathbb{R}^{n+1}\right), \quad t \in \mathbb{R}_{+}, x \in \mathbb{R}^{n}
$$


it is sufficient to find a continuous positive definite function $V(\cdot)$ such that for any solution $x(t)$ of the system (35) the function $V(x(t))$ is decreasing and tending to zero for $t \rightarrow+\infty$. The existence of such function guarantees asymptotic stability of the origin of the system (35) due to Zubov's theorem (see [26] [40]).

If the function $V(x)$ is continuously differentiable then the required monotonicity property can be rewritten in the form of the classical condition [17]:

$$
\dot{V}(x)=\nabla^{T} V(x) f(t, x)<0 .
$$

The inequality (36) is very usable, since it does not require knowing the solutions of (35) in order to check the asymptotic stability. From the practical point of view, it is important to represent monotonicity conditions in the form of differential or algebraic inequalities like (36).

Analysis of sliding mode systems is frequently based on non-smooth or even discontinuous Lyapunov functions [13, 27, 45, 20, 24], which require consideration of generalized derivatives and generalized gradients in order to verify stability conditions. This section presents all necessary backgrounds for the corresponding non-smooth analysis.

\subsection{Derivative Numbers and Monotonicity}

Let $\mathcal{I}$ be one of the following intervals: $[a, b],(a, b),[a, b)$ or $(a, b]$, where $a, b \in \bar{R}, a<b$.

The function $\varphi: \mathbb{R} \rightarrow \mathbb{R}$ is called decreasing on $\mathcal{I}$ iff

$$
\forall t_{1}, t_{2} \in \mathcal{I}: t_{1} \leq t_{2} \Rightarrow \varphi\left(t_{1}\right) \geq \varphi\left(t_{2}\right)
$$

Let $\mathbb{K}$ be a set of all sequences of real numbers converging to zero, i.e.

$$
\left\{h_{n}\right\} \in \mathbb{K} \quad \Leftrightarrow \quad h_{n} \rightarrow 0, h_{n} \neq 0 .
$$

Let a real-valued function $\varphi: \mathbb{R} \rightarrow \mathbb{R}$ be defined on $\mathcal{I}$.

Definition 17 ([46], page 207). A number

$$
D_{\left\{h_{n}\right\}} \varphi(t)=\lim _{n \rightarrow+\infty} \frac{\varphi\left(t+h_{n}\right)-\varphi(t)}{h_{n}}, \quad\left\{h_{n}\right\} \in \mathbb{K}: t+h_{n} \in \mathcal{I}
$$


is called derivative number of the function $\varphi(t)$ at a point $t \in \mathcal{I}$, if finite or infinite limit exists.

The set of all derivative numbers of the function $\varphi(t)$ at a point $t \in \mathcal{I}$ is called contingent derivative:

$$
D_{\mathbb{K}} \varphi(t)=\bigcup_{\left\{h_{n}\right\} \in \mathbb{K}}\left\{D_{\left\{h_{n}\right\}} \varphi(t)\right\} \subseteq \overline{\mathbb{R}} .
$$

A contingent derivative of a vector-valued function $\varphi: \mathbb{R} \rightarrow \mathbb{R}^{n}$ can be defined in the same way. If a function $\varphi(t)$ is differentiable at a point $t \in \mathcal{I}$ then $D_{\mathbb{K}} \varphi(t)=\{\dot{\varphi}(t)\}$.

Lemma 2 ([46], page 208). If a function $\varphi: \mathbb{R} \rightarrow \mathbb{R}$ is defined on $\mathcal{I}$ then

1) the set $D_{\mathbb{K}} \varphi(t) \subseteq \overline{\mathbb{R}}$ is nonempty for any $t \in \mathcal{I}$;

2) for any $t \in \mathcal{I}$ and for any sequence $\left\{h_{n}\right\} \in \mathbb{K}: t+\left\{h_{n}\right\} \in \mathcal{I}$ there exists a subsequence $\left\{h_{n^{\prime}}\right\} \subseteq\left\{h_{n}\right\}$ such that finite or infinite derivative number $D_{\left\{h_{n^{\prime}}\right\}} \varphi(t)$ exists.

Remark, Lemma 2 remains true for a vector-valued function $\varphi: \mathbb{R} \rightarrow \mathbb{R}^{n}$.

Inequalities $y<0, y \leq 0, y>0, y \geq 0$ for $y \in \mathbb{R}^{n}$ are understood in a componentwise sense. If for $\forall y \in D_{\mathbb{K}} \varphi(t)$ we have $y<0$ then we write $D_{\mathbb{K}} \varphi(t)<0$. Other ordering relations $\leq,>, \geq$ for contingent derivatives are interpreted analogously.

The contingent derivative also helps to prove monotonicity of a non-differentiable function.

Lemma 3 ([46], page 266). If a function $\varphi: \mathbb{R} \rightarrow \mathbb{R}$ is defined on $\mathcal{I}$ and the inequality $D_{\mathbb{K}} \varphi(t) \leq 0$ holds for all $t \in \mathcal{I}$, then $\varphi(t)$ is decreasing function on $\mathcal{I}$ and differentiable almost everywhere on $\mathcal{I}$.

Lemma 3 require neither the continuity of the function $\varphi(t)$ nor the finiteness of its derivative numbers. It gives a background for the discontinuous Lyapunov function method. 
Example 9. The function $\varphi(t)=-t-\operatorname{sign}_{\sigma}[t]$ has a negative contingent derivative for all $t \in \mathbb{R}$ and for any $\sigma \in[-1,1]$, where the function $\operatorname{sign}_{\sigma}$ is defined by (1). Indeed, $D_{\mathbb{K}} \varphi(t)=\{-1\}$ for $t \neq 0, D_{\mathbb{K}} \varphi(0)=\{-\infty\}$ if $\sigma \in(-1,1)$ and $D_{\mathbb{K}} \varphi(0)=\{-\infty,-1\}$ if $\sigma \in\{-1,1\}$.

The next lemma simplifies the monotonicity analysis of nonnegative functions.

Lemma 4. If 1) the function $\varphi: \mathbb{R} \rightarrow \mathbb{R}$ is nonnegative on $\mathcal{I}$;

2) the inequality $D_{\mathbb{K}} \varphi(t) \leq 0$ holds for $t \in \mathcal{I}: \varphi(t) \neq 0$;

3) the function $\varphi(t)$ is continuous at any $t \in \mathcal{I}: \varphi(t)=0$;

then $\varphi(t)$ is decreasing function on $\mathcal{I}$ and differentiable almost everywhere on $\mathcal{I}$.

Proof. Suppose the contrary: $\exists t_{1}, t_{2} \in \mathcal{I}: t_{1}<t_{2}$ and $0 \leq \varphi\left(t_{1}\right)<\varphi\left(t_{2}\right)$.

If $\varphi\left(t_{0}\right) \neq 0$ for all $t \in\left[t_{1}, t_{2}\right]$ then Lemma 3 implies that the function $\varphi(t)$ is decreasing on $\left[t_{1}, t_{2}\right]$ and $\varphi\left(t_{1}\right) \geq \varphi\left(t_{2}\right)$.

If there exists $t_{0} \in\left[t_{1}, t_{2}\right]$ such that $\varphi\left(t_{0}\right)=0$ and $\varphi(t)>0$ for all $t \in\left(t_{0}, t_{2}\right]$ then Lemma 3 guarantees that the function $\varphi(t)$ is decreasing on $\left(t_{0}, t_{2}\right]$. Taking into account the condition 3) we obtain the contradiction $\varphi\left(t_{2}\right) \leq \varphi\left(t_{0}\right)=0$.

Finally, let there exists a point $t^{*} \in\left(t_{1}, t_{2}\right]$ such that $\varphi\left(t^{*}\right)>0$ and any neighborhood of the point $t^{*}$ contains a point $t_{0} \in\left[t_{1}, t^{*}\right]: \varphi\left(t_{0}\right)=0$. In this case, let us select the sequence $h_{n}=t_{n}-t^{*}<0$ such that $\varphi\left(t_{n}\right)=0$ and $t_{n} \rightarrow t^{*}$ as $n \rightarrow \infty$. For this sequence we obviously have

$$
D_{\left\{h_{n}\right\}} \varphi\left(t_{1}\right)=\lim _{n \rightarrow \infty} \frac{\varphi\left(t^{*}+h_{n}\right)-\varphi\left(t^{*}\right)}{h_{n}}=\lim _{n \rightarrow \infty} \frac{-\varphi\left(t^{*}\right)}{h_{n}}=+\infty .
$$

This contradicts to the condition 2).

Absolutely continuous functions are differentiable almost everywhere. Monotonicity conditions for them are less restrictive.

Lemma 5 ([47], page 13). If a function $\varphi: \mathbb{R} \rightarrow \mathbb{R}$ defined on $\mathcal{I}$ is absolutely continuous and $\dot{\varphi}(t) \leq 0$ almost everywhere on $\mathcal{I}$ then $\varphi(t)$ is decreasing function on $\mathcal{I}$. 
Lemma below shows relations between solutions of a differential inclusion (27) and its contingent derivatives.

Lemma 6 ([7], page 70). Let a set-valued function $F: \mathbb{R}^{n+1} \rightarrow 2^{\mathbb{R}^{n}}$ be defined, upper-semicontinuous on a closed nonempty set $\Omega \in \mathbb{R}^{n+1}$ and the set $F(t, x)$ be nonempty, compact and convex for all $(t, x) \in \Omega$.

Let an absolutely continuous function $x: \mathbb{R} \rightarrow \mathbb{R}^{n}$ be defined on $\mathcal{I}$ and $(t, x(t)) \in \Omega$ if $t \in \mathcal{I}$. Then

$$
\left.\begin{array}{c}
\dot{x}(t) \in F(t, x(t)) \\
\text { almost everywhere on } \mathcal{I}
\end{array}\right\} \Leftrightarrow \begin{gathered}
D_{\mathbb{K}} x(t) \subseteq F(t, x(t)) \\
\text { everywhere on } \mathcal{I} \text {. }
\end{gathered}
$$

\subsection{Dini derivatives and comparison systems}

The generalized derivatives presented above are closely related with wellknown Dini derivatives (see, for example, [47]).

- Right-hand Upper Dini derivative:

$$
D^{+} \varphi(t)=\limsup _{h \rightarrow 0^{+}} \frac{\varphi(t+h)-\varphi(t)}{h} .
$$

- Right-hand Lower Dini derivative:

$$
D_{+} \varphi(t)=\liminf _{h \rightarrow 0^{+}} \frac{\varphi(t+h)-\varphi(t)}{h} .
$$

- Left-hand Upper Dini derivative:

$$
D^{-} \varphi(t)=\limsup _{h \rightarrow 0^{-}} \frac{\varphi(t+h)-\varphi(t)}{h} .
$$

- Left-hand Lower Dini derivative:

$$
D_{-} \varphi(t)=\liminf _{h \rightarrow 0^{-}} \frac{\varphi(t+h)-\varphi(t)}{h} .
$$

Obviously, $D_{+} \varphi(t) \leq D^{+} \varphi(t)$ and $D_{-} \varphi(t) \leq D^{-} \varphi(t)$. Moreover, definitions of limsup and liminf directly imply that all Dini derivatives belong to the set $D_{\mathbb{K}} \varphi(t)$ and

$$
D_{\mathbb{K}} \varphi(t) \leq 0 \quad \Leftrightarrow \quad\left\{\begin{array}{l}
D^{-} \varphi(t) \leq 0 \\
D^{+} \varphi(t) \leq 0
\end{array}\right.
$$




$$
D_{\mathbb{K}} \varphi(t) \geq 0 \quad \Leftrightarrow \quad\left\{\begin{array}{c}
D_{-} \varphi(t) \geq 0, \\
D_{+} \varphi(t) \geq 0 .
\end{array}\right.
$$

Therefore, all further results for contingent derivative can be rewritten in terms of Dini derivatives.

Theorem 5 (Denjoy-Young-Saks Theorem, [48], page 65). If $\varphi: \mathbb{R} \rightarrow$ $\mathbb{R}$ is a function defined on an interval $\mathcal{I}$, then for almost all $t \in \mathcal{I}$ Dini derivatives of $\varphi(t)$ satisfy one of the following four conditions:

- $\varphi(t)$ has a finite derivative;

- $D^{+} \varphi(t)=D_{-} \varphi(t)$ is finite and $D^{-} \varphi(t)=+\infty, D_{+} \varphi(t)=-\infty$;

- $D^{-} \varphi(t)=D_{+} \varphi(t)$ is finite and $D^{+} \varphi(t)=+\infty, D_{-} \varphi(t)=-\infty$;

- $D^{-} \varphi(t)=D^{+} \varphi(t)=+\infty, D_{-} \varphi(t)=D_{+} \varphi(t)=-\infty$.

This theorem has the following simple corollary, which is important for some further considerations.

Corollary 1. If $\varphi: \mathbb{R} \rightarrow \mathbb{R}$ is a function defined on $\mathcal{I}$, then the equality $D_{\mathbb{K}} \varphi(t)=\{-\infty\}\left(D_{\mathbb{K}} \varphi(t)=\{+\infty\}\right)$ may hold only on a set $\Delta \subseteq \mathcal{I}$ of measure zero.

Consider the system

$$
\dot{y}=g(t, y), \quad(t, y) \in \mathbb{R}^{2}, \quad g: \mathbb{R}^{2} \rightarrow \mathbb{R}
$$

where a function $g(t, y)$ is continuous and defined on a set $\mathbf{G}=(a, b) \times\left(y_{1}, y_{2}\right)$, $a, b, y_{1}, y_{2} \in \mathbb{R}: a<b, y_{1}<y_{2}$. In this case the system (37) has the so-called right-hand maximum solutions for any initial condition $y\left(t_{0}\right)=y_{0},\left(t_{0}, y_{0}\right) \in \mathbf{G}$ (see [47], Remark 9.1, page 25).

Definition 18. A solution $y^{*}\left(t, t_{0}, y_{0}\right)$ of the system (37) with initial conditions $y\left(t_{0}\right)=y_{0},\left(t_{0}, y_{0}\right) \in \boldsymbol{G}$ is said to be right-hand maximum if any other solution $y\left(t, t_{0}, y_{0}\right)$ of the system (37) with the same initial condition satisfies the inequality

$$
y\left(t, t_{0}, y_{0}\right) \leq y^{*}\left(t, t_{0}, y_{0}\right)
$$


for all $t \in \mathcal{I}$, where $\mathcal{I}$ is a time interval on which all solutions exist.

Now we can formulate the following comparison theorem.

Theorem 6 ([47], page 25). Let

1) the right-hand side of the equation (37) be continuous in a region $\boldsymbol{G}$;

2) $y^{*}\left(t, t_{0}, y_{0}\right)$ be the right-hand maximum solution of (37) with the initial condition $y\left(t_{0}\right)=y_{0},\left(t_{0}, y_{0}\right) \in \boldsymbol{G}$, which is defined on $\left[t_{0}, t_{0}+\alpha\right), \alpha \in \mathbb{R}_{+}$;

3) a function $V: \mathbb{R} \rightarrow \mathbb{R}$ be defined and continuous on $\left[t_{0}, t_{0}+\beta\right), \beta \in \mathbb{R}_{+}$, $(t, V(t)) \in \boldsymbol{G}$ for $t \in\left[t_{0}, t_{0}+\beta\right)$ and

$$
V\left(t_{0}\right) \leq y_{0}, \quad D^{+} V(t) \leq g(t, V(t)) \quad \text { for } \quad t \in\left(t_{0}, t_{0}+\beta\right),
$$

then

$$
V(t) \leq y^{*}\left(t, t_{0}, y_{0}\right) \quad \text { for } \quad t \in\left[t_{0}, t_{0}+\min \{\alpha, \beta\}\right) .
$$

Theorem 6 remains true if Dini derivative $D^{+}$is replaced with some other derivative $D_{+}, D^{-}, D_{-}$or $D_{\mathbb{K}}$ (see [47], Remark 2.2, page 11 ).

5.3. Generalized directional derivatives of continuous and discontinuous functions

Stability analysis based on Lyapunov functions requires calculation of derivatives of positive definite functions along trajectories of a dynamic system. If Lyapunov function is non-differentiable, a concept of generalized directional derivatives (see, for example, $[28,49,50]$ ) can be used for this analysis. This survey introduces generalized directional derivatives by analogy with contingent derivatives for scalar functions.

Let $\mathbb{M}(d)$ be a set of all sequences of real vectors converging to $d \in \mathbb{R}^{n}$, i.e.

$$
\left\{v_{n}\right\} \in \mathbb{M}(d) \quad \Leftrightarrow \quad v_{n} \rightarrow d, v_{n} \in \mathbb{R}^{n} .
$$

Let a function $V: \mathbb{R}^{n} \rightarrow \mathbb{R}$ be defined on an open nonempty set $\Omega \subseteq \mathbb{R}^{n}$ and $d \in \mathbb{R}^{n}$.

Definition 19. A number

$$
\begin{gathered}
D_{\left\{h_{n}\right\},\left\{v_{n}\right\}} V(x, d)=\lim _{n \rightarrow+\infty} \frac{V\left(x+h_{n} v_{n}\right)-V(x)}{h_{n}}, \\
\left\{h_{n}\right\} \in \mathbb{K},\left\{v_{n}\right\} \in \mathbb{M}(d): x+h_{n} v_{n} \in \Omega
\end{gathered}
$$


is called directional derivative number of the function $V(x)$ at the point $x \in \Omega$ on the direction $d \in \mathbb{R}^{n}$, if finite or infinite limit exists.

The set of all directional derivative numbers of the function $V(x)$ at the point $x \in \Omega$ on the direction $d \in \mathbb{R}^{n}$ is called directional contingent derivative:

$$
D_{\mathbb{K}, \mathbb{M}(d)} V(x)=\bigcup_{\left\{h_{n}\right\} \in \mathbb{K},\left\{v_{n}\right\} \in \mathbb{M}(d)}\left\{D_{\left\{h_{n}\right\},\left\{v_{n}\right\}} V(x, d)\right\} .
$$

Similarly to Lemma 2 it can be shown that if $x \in \Omega$ then the set $D_{\mathbb{K}, \mathbb{M}(d)} V(x)$ is nonempty for any function $V$ defined on an open nonempty set $\Omega \subseteq \mathbb{R}^{n}$ and any $d \in \mathbb{R}^{n}$. A chain rule for the introduced contingent derivative is described by the following lemma.

Lemma 7. Let a function $V: \mathbb{R}^{n} \rightarrow \mathbb{R}$ be defined on an open nonempty set $\Omega \subseteq \mathbb{R}^{n}$ and a function $x: \mathbb{R} \rightarrow \mathbb{R}^{n}$ be defined on $\mathcal{I}$, such that $x(t) \in \Omega$ if $t \in \mathcal{I}$ and the contingent derivative $D_{\mathbb{K}} x(t) \subseteq \mathbb{R}^{n}$ is bounded for all $t \in \mathcal{I}$.

Then the inclusion

$$
D_{\mathbb{K}} V(x(t)) \subseteq \bigcup_{d \in D_{\mathbb{K}} x(t)} D_{\mathbb{K}, \mathbb{M}(d)} V(x)
$$

holds for all $t \in \mathcal{I}$.

Proof. Since $x(t) \in \Omega$ for $t \in \mathcal{I}$ then Lemma 2 implies that $D_{\mathbb{K}} V(x(t))$ is nonempty for any $t \in \mathcal{I}$. Let $D_{\left\{h_{n}\right\}} V(x(t)) \in D_{\mathbb{K}} V(x(t))$ be an arbitrary derivative number, i.e. by Definition 17 the finite or infinite limit

$$
\lim _{n \rightarrow \infty} \frac{V\left(x\left(t+h_{n}\right)\right)-V(x(t))}{h_{n}}, \quad\left\{h_{n}\right\} \in \mathbb{K}: t+h_{n} \in \mathcal{I}
$$

exists.

Consider now the sequence:

$$
v_{n}=\frac{x\left(t+h_{n}\right)-x(t)}{h_{n}} .
$$

Lemma 2 and inequality $\left|D_{\mathbb{K}} x(t)\right|<+\infty$ implies that there exist finite $d \in$ $D_{\mathbb{K}} x(t)$ and a subsequence $\left\{h_{n^{\prime}}\right\}$ of the sequence $\left\{h_{n}\right\}$ such that $v_{n^{\prime}} \rightarrow d$. Hence,

$D_{\left\{h_{n}\right\}} V(x(t))=\lim _{n \rightarrow \infty} \frac{V\left(x\left(t+h_{n}\right)\right)-V(x(t))}{h_{n}}=\lim _{n^{\prime} \rightarrow \infty} \frac{V\left(x\left(t+h_{n^{\prime}}\right)\right)-V(x(t))}{h_{n^{\prime}}}=$ 


$$
\lim _{n^{\prime} \rightarrow \infty} \frac{V\left(x(t)+h_{n^{\prime}} v_{n^{\prime}}\right)-V(x(t))}{h_{n^{\prime}}}=D_{\left\{h_{n}^{\prime}\right\},\left\{v_{n}^{\prime}\right\}} V(x) .
$$

The proven lemma together with Lemmas 6 and 4 imply the following corollary, which is useful for a non-smooth Lyapunov analysis.

Corollary 2. Let a set-valued function $F: \mathbb{R}^{n+1} \rightarrow 2^{\mathbb{R}^{n}}$ be defined and uppersemicontinuous on $\mathcal{I} \times \Omega$ and the set $F(t, x)$ be nonempty, compact and convex for any $(t, x) \in \mathcal{I} \times \Omega$, where $\Omega \subseteq \mathbb{R}^{n}$ is an open nonempty set.

Let $x\left(t, t_{0}, x_{0}\right)$ be an arbitrary solution of Cauchy problem (27), (31) defined on $\left[t_{0}, t_{0}+\alpha\right)$, where $t_{0} \in \mathcal{I}, x_{0} \in \Omega$ and $\alpha \in \mathbb{R}_{+}$. Let a function $V: \mathbb{R}^{n} \rightarrow \mathbb{R}$ be nonnegative on $\Omega$.

If the inequality $D_{F(t, x)} V(x) \leq 0$ holds for every $t \in \mathcal{I}$ and every $x \in \Omega$ : $V(x) \neq 0$ then the function of time $V\left(x\left(t, t_{0}, x_{0}\right)\right)$ is decreasing on $\left[t_{0}, t_{0}+\alpha\right)$, where

$$
D_{F(t, x)} V(x)=\bigcup_{d \in F(t, x)} D_{\mathbb{K}, \mathbb{M}(d)} V(x)
$$

\subsection{Clarke's gradient of Lipschitz continuous functions}

Let a function $V: \mathbb{R}^{n} \rightarrow \mathbb{R}$ be defined and Lipschitz continuous on an open nonempty set. Then, by Rademacher theorem [51], its gradient exists almost everywhere on $\Omega$ and for each $x \in \Omega$ the following set can be constructed:

$$
\nabla_{C} V(x)=\mathrm{co} \bigcup_{\left\{x_{k}\right\} \in \mathbb{M}(x): \exists \nabla V\left(x_{k}\right)}\left\{\lim _{x_{k} \rightarrow x} \nabla V\left(x_{k}\right)\right\},
$$

which is called the Clarke's generalized gradient of the function $V(x)$ at the point $x \in \Omega$. The set $\nabla_{C} V(x)$ is nonempty, convex and compact for any $x \in \Omega$ and the set-valued mapping $\nabla_{C} V: \mathbb{R}^{n} \rightarrow 2^{\mathbb{R}^{n}}$ is upper-semicontinuous on $\Omega$ (see [50], Proposition 2.6.2, page 70).

The formula (39) gives a procedure for calculation of the generalized gradient of a function. The next lemma presents a chain rule for the Clarke's generalized gradient. 
Lemma 8 ([52], Theorem 2, page 336). Let a Lipschitz continuous function $V: \mathbb{R}^{n} \rightarrow \mathbb{R}$ be defined in an open nonempty set $\Omega \subseteq \mathbb{R}^{n}$ and a absolutely continuous function $x: \mathbb{R} \rightarrow \mathbb{R}^{n}$ be defined on $\mathcal{I}$ such that $x(t) \in \Omega$ for every $t \in \mathcal{I}$.

Then there exists a function $p: \mathbb{R} \rightarrow \mathbb{R}^{n}$ defined on $\mathcal{I}$ such that $p(t) \in$ $\nabla_{C} V(x(t))$ and $\dot{V}(x(t))=p^{T}(t) \dot{x}(t)$ almost everywhere on $\mathcal{I}$.

Lemmas 8 and 5 implies the following corollary.

Corollary 3. Let a set-valued function $F: \mathbb{R}^{n+1} \rightarrow 2^{\mathbb{R}^{n}}$ be defined and uppersemicontinuous on $\mathcal{I} \times \Omega$ and a set $F(t, x)$ be nonempty, compact and convex for any $(t, x) \in \mathcal{I} \times \Omega$, where $\Omega \subseteq \mathbb{R}^{n}$ is an open nonempty set. Let $x\left(t, t_{0}, x_{0}\right)$ be an arbitrary solution of Cauchy problem (27), (31) defined on $\left[t_{0}, t_{0}+\alpha\right)$, where $t_{0} \in \mathcal{I}, x_{0} \in \Omega$ and $\alpha \in \mathbb{R}_{+}$. Let a function $V: \mathbb{R}^{n} \rightarrow \mathbb{R}$ be defined and Lipschitz continuous on $\Omega$.

If the inequality $D_{F(t, x)}^{C} V(x) \leq 0$ holds almost everywhere on $\mathcal{I}$ for every $x \in \Omega$ then the function of time $V\left(x\left(t, t_{0}, x_{0}\right)\right)$ is decreasing on $\left[t_{0}, t_{0}+\alpha\right)$, where

$$
D_{F(t, x)}^{C} V(x)=\bigcup_{d \in F(t, x)} \bigcup_{p \in \nabla_{C} V(x)}\left\{p^{T} d\right\}
$$

If the function $V: \mathbb{R}^{n} \rightarrow \mathbb{R}$ is continuously differentiable then the usual total derivative

$$
\dot{V}_{F(t, x)}(x)=\bigcup_{d \in F(t, x)}\left\{\nabla^{T} V(x) d\right\}
$$

can be used for monotonicity analysis instead of Clarke's or contingent derivative. In this case we have $D_{F(t, x)} V(x)=D_{F(t, x)}^{C} V(x)=\dot{V}_{F(t, x)}(x)$.

\section{Lyapunov function method and convergence rate}

Lyapunov function method is a very effective tool for analysis and design of both linear and nonlinear control systems [19]. Initially, the method was presented for "unrated" (Lyapunov and asymptotic) stability analysis [17]. A development of control theory had required to study a convergence rate together with a stability properties of a control system. This section observes 
the most important achievements of the Lyapunov function method related to a convergence rate estimation of sliding mode systems.

6.1. Analysis of Lyapunov, asymptotic and exponential stability

The continuous function $W: \mathbb{R}^{n} \rightarrow \mathbb{R}$ defined on $\mathbb{R}^{n}$ is said to be positive definite iff $W(0)=0$ and $W(x)>0$ for $x \in \mathbb{R}^{n} \backslash\{0\}$.

Definition 20. A function $V: \mathbb{R}^{n} \rightarrow \mathbb{R}$ is said to be proper on an open nonempty set $\Omega \subseteq \mathbb{R}^{n}: 0 \in \operatorname{int}(\Omega)$ iff

1) it is defined on $\Omega$ and continuous at the origin;

2) there exists a continuous positive definite function $\underline{V}: \mathbb{R}^{n} \rightarrow \mathbb{R}$ such that

$$
\underline{V}(x) \leq V(x) \quad \text { for } \quad x \in \Omega .
$$

A positive definite function $W: \mathbb{R}^{n} \rightarrow \mathbb{R}$ is called radially unbounded if $W(x) \rightarrow+\infty$ for $\|x\| \rightarrow+\infty$.

Definition 21. A function $V: \mathbb{R}^{n} \rightarrow \mathbb{R}$ is said to be globally proper iff it is proper on $\mathbb{R}^{n}$ and the positive definite function $\underline{V}: \mathbb{R}^{n} \rightarrow \mathbb{R}$ is radially unbounded.

If $V$ is continuous on $\Omega$, then $\underline{V}(x)=V(x)$ for $x \in \Omega$ and Definition 21 corresponds to the usual notion of proper positive definite function (see, for example, [44]).

For a given number $r \in \mathbb{R}$ and a given positive definite function $W: \mathbb{R}^{n} \rightarrow \mathbb{R}$ defined on $\Omega$ let us introduce the set

$$
\Pi(W, r)=\{x \in \Omega: W(x)<r\}
$$

which is called the level set of the function $W$.

Theorems on Lyapunov and asymptotic stability given below are obtained by a combination of Zubov's theorems (see, for example, [40], pages 566-568) with Corollary 2. 
Theorem 7. Let a function $V: \mathbb{R}^{n} \rightarrow \mathbb{R}$ be proper on an open nonempty set $\Omega \subseteq \mathbb{R}^{n}: 0 \in \operatorname{int}(\Omega)$ and

$$
D_{F(t, x)} V(x) \leq 0 \quad \text { for } \quad t \in \mathbb{R} \text { and } x \in \Omega \backslash\{0\} .
$$

Then the origin of the system (27) is Lyapunov stable.

Proof. Since $V(x)$ is proper, then there exist continuous positive definite function $\underline{V}(x)$ such that $\underline{V}(x) \leq V(x)$ for all $x \in \Omega$.

Let $h=\sup _{r \in \mathbb{R}_{+}: \mathcal{B}(r) \subseteq \Omega} r$ and $\lambda(\varepsilon)=\inf _{x \in \mathbb{R}^{n}:\|x\|=\varepsilon} \underline{V}(x)>0$, where $\varepsilon \in(0, h]$.

The function $V(x)$ is continuous at the origin, so $\exists \delta \in(0, \varepsilon): V(x)<\lambda(\varepsilon)$ if $x \in \mathcal{B}(\delta)$. Moreover, $\mathcal{B}(\delta) \subseteq \mathcal{U}(\varepsilon)=\Pi(V, \lambda(\varepsilon)) \cap \mathcal{B}(\varepsilon)$.

Let $t_{0} \in \mathbb{R}$ and $x_{0} \in \mathcal{U}(\varepsilon)$ (in partial case $x_{0} \in \mathcal{B}(\delta)$ ). The system (27) satisfies Theorem 3 and it has solutions, which can be continued up to the boundary of $\Omega$. Consider an arbitrary solution $x\left(t, t_{0}, x_{0}\right)$ of (27). The inequality (42) and Corollary 2 implies that the function of time $V\left(x\left(t, t_{0}, x_{0}\right)\right)$ is decreasing for $t>t_{0}$, i.e. $V\left(x\left(t, t_{0}, x_{0}\right)\right) \leq V\left(x_{0}\right)<\lambda(\varepsilon)$.

In this case, $x\left(t, t_{0}, x_{0}\right) \in \mathcal{B}(\varepsilon)$ for $t>t_{0}$. Indeed, otherwise there exists $t^{*}>t_{0}:\left\|x\left(t^{*}, t_{0}, x_{0}\right)\right\|=\varepsilon$, so $V\left(x\left(t^{*}, t_{0}, x_{0}\right)\right) \geq \underline{V}\left(x\left(t^{*}, t_{0}, x_{0}\right)\right) \geq \lambda(\varepsilon)$.

The proven property also implies that even if a solution of (27) with $t_{0} \in \mathbb{R}$ and $x_{0} \in \mathcal{U}(\varepsilon)$ was initially defined on finite interval $\left[t_{0}, t_{0}+\alpha\right), \alpha \in \mathbb{R}_{+}$, it can be prolonged for all $t>t_{0}$.

Asymptotic stability requires analysis of an attraction set. Lyapunov function approach may provide an estimate of this set.

Theorem 8. Let a function $V: \mathbb{R}^{n} \rightarrow \mathbb{R}$ be proper on an open nonempty set $\Omega \subseteq \mathbb{R}^{n}: 0 \in \operatorname{int}(\Omega)$, a function $W: \mathbb{R}^{n} \rightarrow \mathbb{R}$ be a continuous positive definite and

$$
D_{F(t, x)} V(x) \leq-W(x) \quad \text { for } \quad t \in \mathbb{R} \text { and } x \in \Omega \backslash\{0\} .
$$

Then the origin of the system (27) is asymptotically stable with an attraction domain

$$
\mathcal{U}=\Pi(V, \lambda(h)) \cap \mathcal{B}(h),
$$


where $\lambda(h)=\inf _{x \in \mathbb{R}^{n}:\|x\|=h} \underline{V}(x)$ and $h \leq \sup _{r \in \mathbb{R}_{+}: \mathcal{B}(r) \subseteq \Omega} r$.

If $V$ is globally proper and $\Omega=\mathbb{R}^{n}$ then the origin of the system (27) is globally asymptotically stable $\left(\mathcal{U}=\mathbb{R}^{n}\right)$.

Proof. Theorem 7 implies that an arbitrary solution $x\left(t, t_{0}, x_{0}\right)$ of $(27)$ with $t_{0} \in \mathbb{R}$ and $x_{0} \in \mathcal{U}(\varepsilon)$ is defined for all $t>t_{0}$ and $x\left(t, t_{0}, x_{0}\right) \in \mathcal{B}(\varepsilon)$, where $\varepsilon \in(0, h]$ and $\mathcal{U}(\varepsilon)=\Pi(V, \lambda(\varepsilon)) \cap \mathcal{B}(\varepsilon)$. Moreover, the function of time $\tilde{V}(t)=$ $V\left(x\left(t, t_{0}, x_{0}\right)\right)$ is decreasing for all $t>t_{0}$. So, in order to prove asymptotic stability we just need to show that $\mu=0$, where $\mu=\inf _{t>t_{0}} \tilde{V}(t)$.

Suppose a contradiction, i.e. $\mu>0$.

The function $V(x)$ is continuous at the origin, so there exists $r>0$ such that $V(x)<\mu$ for all $x \in \mathcal{B}(r)$. Since $\mu>0$ then $x\left(t, t_{0}, x_{0}\right) \notin \mathcal{B}(r)$ for all $t>t_{0}$.

Introduce the following compact set $\Theta=\left\{x \in \mathbb{R}^{n}: r \leq\|x\| \leq \varepsilon\right\}$. Since $W(x)$ is continuous and positive definite, then we have $W_{0}=\inf _{x \in \Theta} W(x)>0$.

The inequality $D_{F(t, x)} V(x) \leq-W(x)$ and the exclusion $x\left(t, t_{0}, x_{0}\right) \notin \mathcal{B}(r)$ imply $D_{\mathbb{K}} \tilde{V}(t) \leq-W_{0}$ for all $t>t_{0}$.

Since $\tilde{V}(t)$ is decreasing then it is differentiable almost everywhere on $\left[t_{0}, t_{0}+\right.$ $\Delta$ ], where $\Delta=V\left(x_{0}\right) / W_{0}$. Hence (see, for example, [53], page 111),

$$
V\left(t_{0}+\Delta\right)-V\left(t_{0}\right) \leq \int_{t_{0}}^{t_{0}+\Delta} \dot{V}(\tau) d \tau \leq-W_{0} \Delta=-V\left(t_{0}\right),
$$

i.e. $V\left(t_{0}+\Delta\right) \leq 0<\mu$. This contradicts our supposition. So, $V\left(x\left(t, t_{0}, x_{0}\right)\right) \rightarrow 0$ or equivalently $x\left(t, t_{0}, x_{0}\right) \rightarrow 0$ if $t \rightarrow+\infty$.

If the function $V$ is globally proper then global asymptotic attractiveness follows from $\lim _{\varepsilon \rightarrow+\infty} \lambda(\varepsilon)=+\infty$ due to radial unboundedness of $\underline{V}$.

Exponential convergence asks for additional properties of Lyapunov functions.

Theorem 9. Let conditions of Theorem 8 hold, the function $V(x)$ is continuous on an open nonempty set $\Omega \subset \mathbb{R}^{n}: 0 \in \operatorname{int}(\Omega)$ and there exist $\alpha, r_{1}, r_{2} \in \mathbb{R}_{+}$:

$$
r_{1}\|x\| \leq V(x) \leq r_{2}\|x\| \text { and } W(x) \geq \alpha V(x)
$$

then the origin of the system (27) is exponentially stable with a rate $\alpha \in \mathbb{R}_{+}$. 
This theorem can be proven by analogy to a classical theorem on exponential stability (see, for example, [19], page 171) using Lemma 6.

The presented theorems shows that discontinuous and non-Lipschitzian Lyapunov functions can also be used for stability analysis. If $V(x)$ is Lipschitz continuous then all theorems on stability can be reformulated using Clarke's gradient.

The following important theorem declares that a smooth Lyapunov function always exists for a time-invariant asymptotically stable differential inclusion (27).

Theorem 10 ([44], Theorem 1.2). Let a set-valued function $F: R^{n} \rightarrow \mathbb{R}^{n}$ be defined and upper-semicontinuous in $\mathbb{R}^{n}$. Let $F(x)$ be nonempty, compact and convex for any $x \in \mathbb{R}^{n}$. If the origin of the system

$$
\dot{x} \in F(x)
$$

is globally uniformly asymptotically stable iff there exists a globally proper function $V(\cdot) \in \mathbb{C}^{\infty}\left(\mathbb{R}^{n}\right)$ and a function $W(\cdot) \in \mathbb{C}^{\infty}\left(\mathbb{R}^{n}\right): W(x)>0$ for $x \neq 0$ such that

$$
\max _{y \in F(x)} \nabla^{T} V(x) y \leq-W(x), \quad x \in \mathbb{R}^{n} \backslash\{0\} .
$$

However, the practice shows that designing of a Lyapunov function for nonlinear and/or discontinuous system is a nontrivial problem even for a two dimensional case. Frequently, in order to analyze stability of a sliding mode control system it is simpler to design a non-smooth Lyapunov function (see, for example, $[3],[20],[24])$.

\subsection{Lyapunov analysis of finite-time stability}

Analysis of finite-time stability using the Lyapunov function method allows us to estimate of a settling time a priori. The proof of the next theorem follows the ideas introduced in [13] and [54]. 
Theorem 11. Let a function $V: \mathbb{R}^{n} \rightarrow \mathbb{R}$ be proper on an open nonempty set $\Omega \subseteq \mathbb{R}^{n}: 0 \in \operatorname{int}(\Omega)$ and

$$
D_{F(t, x)} V(x) \leq-1 \quad \text { for } \quad t \in \mathbb{R} \text { and } x \in \Omega \backslash\{0\} .
$$

Then the origin of the system (27) is finite-time stable with an attraction domain $\mathcal{U}$ defined by (43) and

$$
T\left(x_{0}\right) \leq V\left(x_{0}\right) \quad \text { for } \quad x_{0} \in \mathcal{U},
$$

where $T(\cdot)$ is a settling-time function.

If a function $V$ is globally proper on $\Omega=\mathbb{R}^{n}$ then the inequality (44) implies global finite-time stability of the system (27).

Proof. Theorem 8 implies that the origin of the system (27) is asymptotically stable with the attraction domain $\mathcal{U}$. This means that any solution $x\left(t, t_{0}, x_{0}\right), x_{0} \in \mathcal{U}$ of the system (27) exists for $\forall t>t_{0}$. Therefore, we need to show finite-time attractivity. Consider the interval $\left[t_{0}, t_{1}\right], t_{1}=t_{0}+V\left(x_{0}\right)$.

Suppose a contradiction: $x\left(t, t_{0}, x_{0}\right) \neq 0$ for $\forall t \in\left[t_{0}, t_{1}\right]$. Denote $\tilde{V}(t)=$ $V\left(x\left(t, t_{0}, x_{0}\right)\right)$. Lemma 7 implies

$$
D_{\mathbb{K}} \tilde{V}(t) \leq D_{F(t, x)} V\left(x\left(t, t_{0}, x_{0}\right)\right) \leq-1, \quad \forall t \in\left[t_{0}, t_{1}\right]
$$

Hence, by Lemma 3 the function $\tilde{V}(t)$ is decreasing on $\left[t_{0}, t_{1}\right]$ and differentiable almost everywhere on $\left[t_{0}, t_{1}\right]$. Then

$$
\tilde{V}\left(t_{1}\right)-\tilde{V}\left(t_{0}\right) \leq \int_{t_{0}}^{t_{1}} \frac{d}{d t} \tilde{V}(\tau) d \tau \leq-\left(t_{1}-t_{0}\right)=-V\left(x_{0}\right)
$$

(see, for example, [53], page 111), i.e. $\tilde{V}\left(t_{1}\right)=V\left(x\left(t_{1}, t_{0}, x_{0}\right)\right) \leq \tilde{V}\left(t_{0}\right)-$ $V\left(x_{0}\right)=V\left(x\left(t_{0}, t_{0}, x_{0}\right)\right)-V\left(x_{0}\right)=0$. Since $V(x)$ is positive definite then $V\left(x\left(t_{1}, t_{0}, x_{0}\right)\right) \leq 0 \Rightarrow V\left(x\left(t_{1}, t_{0}, x_{0}\right)\right)=0 \Leftrightarrow x\left(t_{1}, t_{0}, x_{0}\right)=0$, i.e. the origin of the system (27) is finite-time attractive with the settling time estimate (45).

Evidently, if under conditions of Theorem 11 there exists a continuous function $\bar{V}: \mathbb{R}^{n} \rightarrow \mathbb{R}$ such that $V(x) \leq \bar{V}(x)$ for $\forall x \in \Omega$ then the origin of the system (27) is uniformly finite-time stable. 
Example 10. Consider again the uniformly finite-time stable system

$$
\dot{x}=-x^{\left[\frac{1}{2}\right]}(1-|x|), x \in \mathbb{R},
$$

and show that its settling-time function

$$
T(x)=\ln \left(\frac{1+|x|^{\frac{1}{2}}}{1-|x|^{\frac{1}{2}}}\right)
$$

satisfies all conditions of Theorem 11. Indeed, it is continuous and proper on $\mathcal{B}(1)$. Finally, it is differentiable for $x \in \mathcal{B}(1) \backslash\{0\}$ and

$$
\dot{T}(x)=\frac{\partial T}{\partial x} \dot{x}=\frac{1}{x^{\left[\frac{1}{2}\right]}(1-|x|)} \dot{x}=-1 \quad \text { for } \quad x \neq 0 .
$$

The last example shows that a settling-time function of finite-time stable system is a Lyapunov function in a generalized sense. Theorem 11 operates with a very large class Lyapunov functions. However, its conditions are still rather conservative. For example, the settling-time function from Example 6 can not be considered as a Lyapunov function candidate, since it is discontinuous at the origin, so it is not proper. However, even proper settling-time functions of sliding mode systems may not satisfy the condition (44).

Example 11. Consider the twisting second order sliding mode system [55]

$$
\left(\begin{array}{c}
\dot{x}_{1} \\
\dot{x}_{2}
\end{array}\right) \in F\left(x_{1}, x_{2}\right)=\left(\begin{array}{c}
y \\
-2 \overline{\operatorname{sign}}\left[x_{1}\right]-\overline{\operatorname{sign}}\left[x_{2}\right]
\end{array}\right),
$$

which is uniformly finite-time stable with the settling-time function [54] :

$$
T_{t w}(x)=p \sqrt{\left|x_{1}\right|+\frac{x_{2}^{2}}{2\left(2+\operatorname{sign}\left[x_{1} x_{2}\right]\right)}}+\frac{\left|x_{2}\right| \operatorname{sign}\left[x_{1} x_{2}\right]}{2+\operatorname{sign}\left[x_{1} x_{2}\right]}, \quad p=\frac{4 \sqrt{2}}{3-\sqrt{3}}
$$

The function $T_{t w}$ is globally proper, Lipschtz continuous outside the origin and continuously differentiable for $x y \neq 0$

$D_{F\left(x_{1}, x_{2}\right)} T_{t w}\left(x_{1}, x_{2}\right)=\frac{\partial T_{t w}}{\partial x_{1}} x_{2}+\frac{\partial T_{t w}}{\partial x_{2}}\left(-2 \operatorname{sign}\left[x_{1}\right]-\operatorname{sign}\left[x_{2}\right]\right)=-1$ for $x_{1} x_{2} \neq 0$.

However, $D_{F\left(x_{1}, x_{2}\right)} T_{t w}\left(x_{1}, x_{2}\right) \bigcap \mathbb{R}_{+} \neq \emptyset$ for $x_{1}=0$. So, $T_{t w}(x, y)$ does not satisfy (44). Applying Clarke's gradient does not help to avoid this problem. 
In the same time, if $x\left(t, t_{0}, x_{0}\right)$ is an arbitrary solution of the system (46), then $D_{\mathbb{K}} T_{t w}\left(x\left(t, t_{0}, x_{0}\right)\right) \leq-1$ for $\forall t>t_{0}: x\left(t, t_{0}, x_{0}\right) \neq 0$ (see [54] for the details).

Remark, if $p>\frac{4 \sqrt{2}}{3-\sqrt{3}}$ then the function $T_{t w}(x)$ satisfies the conditions of Theorem 11 and $D_{F\left(x_{1}, x_{2}\right)} T_{t w}(x)=\{-\infty\}$ for $x_{1}=0$.

Sometimes the less restrictive finite-time stability condition

$$
\begin{gathered}
D_{\mathbb{K}} V\left(x\left(t, t_{0}, x_{0}\right)\right) \leq-1, \quad t \geq t_{0}: x\left(t, t_{0}, x_{0}\right) \neq 0, \\
x\left(t, t_{0}, x_{0}\right) \in \Phi\left(t_{0}, x_{0}\right), t_{0} \in \mathbb{R}, x_{0} \in \mathcal{U} .
\end{gathered}
$$

has to be considered instead of (44). Examples of applying the condition (47) for analysis of second order sliding mode systems can be found in [54], [22]. They demonstrate that frequently we do not need to know a solution $x\left(t, t_{0}, x_{0}\right)$ of (27) in order to check the condition (47).

Example 12. Consider the system

$$
\dot{x}=-\frac{\left(2-\operatorname{sign}\left[x_{1} x_{2}\right]\right)}{\|x\|} x, \quad x=\left(x_{1}, x_{2}\right)^{T} \in \mathbb{R}^{2} .
$$

It is uniformly finite-time stable. Its settling time function is discontinuous

$$
T(x)=\left\{\begin{array}{ccc}
\|x\| & \text { for } & x_{1} x_{2} \geq 0 \\
\frac{1}{3}\|x\| & \text { for } & x_{1} x_{2}<0
\end{array}\right.
$$

However, the function $T(x)$ is the generalized Lyapunov function, since it is globally proper and

$$
D_{\mathbb{K}} T\left(x\left(t, t_{0}, x_{0}\right)\right)=-1 \quad \text { for } t>t_{0}: x\left(t, t_{0}, x_{0}\right) \neq 0,
$$

where $x\left(t, t_{0}, x_{0}\right) \in \Phi\left(t_{0}, x_{0}\right), t_{0} \in \mathbb{R}$ and $x_{0} \in \mathbb{R}^{2}$.

Theorem $12\left([14]\right.$, Theorem 4.2). Let a continuous function $V: \mathbb{R}^{n} \rightarrow \mathbb{R}$ be proper on an open nonempty set $\Omega \subseteq \mathbb{R}^{n}: 0 \in \operatorname{int}(\Omega)$ and

$$
D_{F(t, x)} V(x) \leq-r V^{\rho}(x), \quad t>t_{0}, x \in \Omega,
$$


where $r \in \mathbb{R}_{+}, 0<\rho<1$. Then the origin of the system (27) is uniformly finite-time stable with an attraction domain $\mathcal{U}$ defined by (43) and the settling time function $T(\cdot)$ is estimated as follows

$$
T\left(x_{0}\right) \leq \frac{V^{1-\rho}\left(x_{0}\right)}{r(1-\rho)} \quad \text { for } \quad x_{0} \in \mathcal{U} .
$$

Proof. Let $x\left(t, t_{0}, x_{0}\right), x_{0} \in \mathcal{U}$ be any solution of $(27)$ and $\tilde{V}(t)=V\left(x\left(t, t_{0}, x_{0}\right)\right)$. Since

$$
D_{\mathbb{K}} \tilde{V}(t) \leq D_{F(t, x)} V\left(x\left(t, t_{0}, x_{0}\right)\right) \leq-r \tilde{V}^{\rho}(t)
$$

(see, Lemma 7) then Lemma 6 implies that $\tilde{V}(t) \leq y(t), t>t_{0}$, where $y(t)$ is a right-hand maximum solution of the following Cauchy problem

$$
\dot{y}(t)=-r y^{\rho}(t), \quad y\left(t_{0}\right)=V\left(x_{0}\right),
$$

i.e.

$$
y(t)=\left\{\begin{array}{cccc}
\left(V\left(x_{0}\right)^{1-\rho}-r(1-\rho)\left(t-t_{0}\right)\right)^{\frac{1}{1-\rho}} & \text { for } & t \in\left[t_{0}, t_{0}+\frac{V^{1-\rho}\left(x_{0}\right)}{r(1-\rho)}\right], \\
0 & \text { for } & t>\frac{V^{1-\rho}\left(x_{0}\right)}{r(1-\rho)} .
\end{array}\right.
$$

This implies $V\left(x\left(t, t_{0}, x_{0}\right)\right)=0$ for $\forall t>\frac{V^{1-\rho}\left(x_{0}\right)}{r(1-\rho)}$.

A global finite-time stability can be analyzed using globally proper Lyapunov functions in Theorems 11 and 12.

Example 13. Consider the so-called super-twisting system [55]

$$
\left(\begin{array}{l}
\dot{x} \\
\dot{y}
\end{array}\right) \in F(x, y)=\left(\begin{array}{c}
-\alpha x^{\left[\frac{1}{2}\right]}+y \\
-\beta \cdot \overline{\operatorname{sign}}[x]
\end{array}\right)
$$

where $x \in \mathbb{R}, y \in \mathbb{R}, \alpha>0, \beta>0$. Recall, $x^{[\mu]}=|x|^{\mu} \operatorname{sign}[x], \mu \in \mathbb{R}_{+}$.

The function [24]

$$
V(x, y)=\left(2 \beta+\alpha^{2} / 2\right)|x|+y^{2}-\alpha y x^{\left[\frac{1}{2}\right]}
$$

is the generalized Lyapunov function for the system (48). Indeed, this function is globally proper and continuous (but not Lipschitz continuous on the line $x=0$ ).

For $x \neq 0$ this function is differentiable and

$$
D V_{F(x, y)}(x, y) \leq-\gamma \sqrt{V(x, y)}
$$


where $\gamma=\gamma(\alpha, \beta)>0$ is a positive number (see [24] for details).

For $x=0$ and $y \neq 0$ we need to calculate a generalized directional derivative. So, consider the limit

$$
D_{\left\{h_{n}\right\},\left\{u_{n}\right\}} V(0, y)=\lim _{n \rightarrow \infty} \frac{V\left(h_{n} u_{n}^{x}, y+h_{n} u_{n}^{y}\right)-V(0, y)}{h_{n}}
$$

where $\left\{h_{n}\right\} \in \mathbb{K}, u_{n}=\left(u_{n}^{x}, u_{n}^{y}\right)^{T},\left\{u_{n}\right\} \in \mathbb{M}(d), d \in F(0, y)$. In this case, $u_{n}^{x} \rightarrow y$ and $u_{n}^{y} \rightarrow q, q \in[-\beta, \beta]$. Hence,

$D_{\left\{h_{n}\right\},\left\{u_{n}\right\}} V(0, y)=\lim _{n \rightarrow \infty} \frac{\left(2 \beta+\alpha^{2} / 2\right)\left|h_{n} y\right|+\left(y+h_{n} q\right)^{2}-\alpha\left(h_{n} y\right)^{\left[\frac{1}{2}\right]}\left(y+h_{n} q\right)-y^{2}}{h_{n}}$.

Obviously, $D_{\left\{h_{n}\right\},\left\{u_{n}\right\}} V(0, y)=-\infty$. Therefore,

$$
D_{F(x, y)} V(0, y)=\{-\infty\} \leq-\gamma \sqrt{V(0, y))} \quad \text { for } \quad y \neq 0
$$

and the super-twisting system is uniformly finite-time stable with the settling time estimate $T(x, y) \leq 2 \sqrt{V(x, y)} / \gamma$.

By Corollary 1, the set of time instants $t>t_{0}: D_{\mathbb{K}} V(x(t), y(t))=\{-\infty\}$ may have only the measure zero. This means that the line $x=0$ for $y \neq 0$ can not be sliding set of the system (48). The sliding mode may appear only at the origin.

\subsection{Fixed-time stability analysis}

Locally fixed-time stability property is very close to finite-time stability, so it can be established using Theorem 11 just including additional condition : $V(x) \leq T_{\max }$ for $\forall x \in \Omega$, where $T_{\max } \in \mathbb{R}_{+}$. An alternative Lyapunov characterization of fixed-time stability can be obtained using the ideas introduced in the proof of Corollary 2.24 from [31].

Theorem 13 ([30], page 2106). Let a continuous function $V: \mathbb{R}^{n} \rightarrow \mathbb{R}$ be proper on an open connected set $\Omega: 0 \in \operatorname{int}(\Omega)$. If for some numbers $\mu \in$ $(0,1), \nu \in \mathbb{R}_{+}, r_{\mu} \in \mathbb{R}_{+}, r_{\nu} \in \mathbb{R}_{+}$the following inequlity

$$
D_{F(t, x)} V(x) \leq\left\{\begin{array}{lll}
-r_{\mu} V^{1-\mu}(x) & \text { for } & x \in \Omega: V(x) \leq 1, \\
-r_{\nu} V^{1+\nu}(x) & \text { for } \quad x \in \Omega: V(x) \geq 1,
\end{array} \quad t>t_{0}, x \in \Omega,\right.
$$


holds, then the origin of the system (27) is fixed-time stable with the attraction domain $\mathcal{U}$ defined by (43) and the maximum settling time is estimated by

$$
T(x) \leq T_{\max } \leq \frac{1}{\mu r_{\mu}}+\frac{1}{\nu r_{\nu}} .
$$

If $\Omega=\mathbb{R}^{n}$ and a function $V$ is radially unbounded then the origin of the system (27) is globally fixed-time stable.

Proof. Theorem 8 implies that the origin of the system (27) is asymptotically stable with the attraction domain $\mathcal{U}$. This means that any solution $x\left(t, t_{0}, x_{0}\right), x_{0} \in \mathcal{U}$ of the system (27) exists for $\forall t>t_{0}$. We just need to proof that the estimate (49) implies fixed-time attractivity.

Indeed, for any trajectory $x\left(t, t_{0}, x_{0}\right)$ of the system (6) with $V\left(x_{0}\right)>1$, there exists a time instant $T_{1}=T_{1}\left(x_{0}\right) \leq \frac{1}{\nu r_{\nu}}: V\left(x\left(T_{1}, t_{0}, x_{0}\right)\right)=1$. On the other hand, for any trajectory $x\left(t, t_{1}, x_{1}\right)$ with $V\left(x_{1}\right) \leq 1$, there exists a time instant $T_{2}=T_{2}\left(x_{1}\right) \leq \frac{1}{\mu r_{\mu}}: V\left(x\left(t, t_{1}, x_{1}\right)\right) \rightarrow 0$ for $t \rightarrow T_{2}$. These facts can be easily proven analogously to Theorem 12 .

This result also can be used for fixed-time stability analysis of high-order sliding mode control systems.

Example 14 ([30], page 2108). Consider the sliding mode control system

$$
\left\{\begin{array}{l}
\dot{x}=y, \\
\dot{y}=u+d(t), \\
u=-\frac{\alpha_{1}+3 \beta_{1} x^{2}+\gamma}{2} \operatorname{sign}[s]-\left(\alpha_{2} s+\beta_{2} s^{3}\right)^{\left[\frac{1}{2}\right]},
\end{array}\right.
$$

where $x \in \mathbb{R}, y \in \mathbb{R},|d(t)|<C, \alpha_{1}, \alpha_{2}, \beta_{1}, \beta_{2}, C \in \mathbb{R}_{+}, \gamma>2 C$ and the switching surface $s=0$ is defined by

$$
s=y+\left(y^{[2]}+\alpha_{1} x+\beta_{1} x^{3}\right)^{\left[\frac{1}{2}\right]} .
$$

The original discontinuous systems corresponds to the following extended differential inclusion:

$$
\left\{\begin{array}{l}
\dot{x}=y, \\
\dot{y} \in\left\{-\frac{\alpha_{1}+3 \beta_{1} x^{2}+\gamma}{2}\right\} \cdot \overline{\operatorname{sign}}[s] \dot{+}\left\{-\left(\alpha_{2} s+\beta_{2} s^{3}\right)^{\left[\frac{3}{2}\right]}\right\} \dot{+}[-C, C] .
\end{array}\right.
$$


Consider the function $V(s)=|s|$ and calculate its generalized derivative along trajectories of the last system

$$
D_{F} V(s) \leq-\left(\alpha_{2} V(s)+\beta_{2} V^{3}(s)\right)^{\frac{1}{2}} \quad \text { for } s \neq 0
$$

(see [30] for the details). This implies that the sliding surface $s=0$ is fixed-time attractive with the estimate of a reaching time:

$$
T_{s} \leq \frac{2}{\sqrt{\alpha_{2}}}+\frac{2}{\sqrt{\beta_{2}}}
$$

The sliding motion equation for $s=0$ has the form

$$
\dot{x}=-\left(\frac{\alpha_{1}}{2} x+\frac{\beta_{1}}{2} x^{3}\right)^{\left[\frac{1}{2}\right]} .
$$

This system is fixed-time stable and a global estimate of the settling-time function $T(x, y)$ for the original system is

$$
T(x, y) \leq T_{\max } \leq \frac{2 \sqrt{2}}{\sqrt{\alpha_{1}}}+\frac{2 \sqrt{2}}{\sqrt{\beta_{1}}}+\frac{2}{\sqrt{\alpha_{2}}}+\frac{2}{\sqrt{\beta_{2}}} .
$$

\section{Conclusions}

The paper surveys mathematical tools required for stability analysis of sliding mode systems. It discusses definitions of solutions for systems with discontinuous right-hand sides, which effectively describe sliding mode systems. It observes an evolution of stability notions, convergence rate properties and underlines differences between finite-time and fixed-time stable systems in local and global cases. The paper considers elements of the theory of generalized derivatives and presents a generalized Lyapunov function method for asymptotic, exponential, finite-time and fixed-time stability analysis of discontinuous systems. Theorems on finite-time and fixed-time stability provide rigorous mathematical justifications of formal applying non-Lipschitz Lyapunov functions presented in $[23,24,25]$ for stability analysis of second order sliding mode systems.

It is worth to stress that the presented tutorial summarize methods required for stability analysis of the so-called "ideal" sliding modes. The practical realization of sliding mode control requires extended analysis, which takes into 
account sampling, hysteresis and delay effects, measurement errors, discretization, etc. Robustness analysis of "real" sliding modes goes out of the scope of this paper. Practical stability analysis of sliding mode systems based on two Lyapunov functions was presented in [56]. Stability of the real coordinates in the sliding mode was studied in [57]. More general approach to robustness analysis of "real" sliding modes based on ISS theory of homogeneous systems can be found in [58].

\section{Acknowledgements}

We would like to thank the editor and the reviewers, who provided valuable comments helping us to improve the presentation of the paper.

\section{References}

[1] Flugge-Lotz I. Discontinuous automatic control. Princeton University Press, 1953.

[2] Tsypkin YZ. Relay Control Systems. Cambridge University Press, 1984.

[3] Utkin VI. Sliding Modes in Control Optimization. Springer-Verlag: Berlin, 1992.

[4] Boiko I. Discontinuous control systems : frequency-domain analysis and design. Boston : Birkhauser, 2009.

[5] Choi S, Hedrick J. Robust throttle control of automotive engines. ASME Journal of Dynamic Systems, Measurement, and Control 1996; 118:92-98.

[6] Utkin V, Guldner J, Shi J. Sliding Mode Control in Electro-Mechanical Systems. CRC Press, 2009.

[7] Filippov AF. Differential Equations with Discontinuous Righthand Sides. Kluwer Academic Publishers, 1988.

[8] Edwards C, Spurgeon S. Sliding Mode Control: Theory and Applications. Taylor and Francis, 1998. 
[9] Neimark YI. Note on A. Filippov paper. Proceedings of 1st IFAC Congress II, Butterworths, London, 1961.

[10] Aizerman MA, Pyatnitskii E. Foundations of theory of discontinuous systems i. Automation and Remote Control 1974; 35:1066-1079.

[11] Bartolini G, Zolezzi T. Variable structure systems nonlinear in the control law. IEEE Transactions on Automatic Control 1985; 30(7):681-684.

[12] Heemels W, Weiland S. Input-to-state stability and interconnections of discontinuous dynamical systems. Automatica 2008; 44:3079-3086.

[13] Roxin E. On finite stability in control systems. Rendiconti del Circolo Matematico di Palermo 1966; 15:273-283.

[14] Bhat S, Bernstein D. Finite time stability of continuous autonomous systems. SIAM J. Control Optim. 2000; 38(3):751-766.

[15] Orlov Y. Finite time stability and robust control synthesis of uncertain switched systems. SIAM J. Control Optim. 2005; 43(4):1253-1271.

[16] Moulay E, Perruquetti W. Finite-time stability conditions for nonautonomous continuous systems. International Journal of Control 2008; 81(5):797-803.

[17] Lyapunov AM. The general problem of the stability of motion. Taylor \& Francis, 1992.

[18] Barbashin EA. Lyapunov Functions. Moscow:Nauka, 1970 (in Russian).

[19] Bacciotti A, Rosier L. Lyapunov Functions and Stability in Control Theory. 2nd edn., Springer, Berlin, 2005.

[20] Polyakov A, Poznyak A. Reaching time estimation for "super-twisting" second order sliding mode controller via Lyapunov function designing. IEEE Transactions on Automatic Control 2009; 54(8):1951-1955. 
[21] Polyakov A, Poznyak A. Lyapunov function design for finite-time convergence analysis: "twisting" controller for second order sliding mode realization. Automatica 2009; 45:444-448.

[22] Poznyak A, Polyakov A, Strygin V. Analysis of finite-time convergence by the method of Lyapunov functions in systems with second order sliding modes. Journal of Applied Mathematics and Mechanics 2011; 75:289-303.

[23] Moreno J. A Lyapunov approach to analysis and design of second order sliding mode algorithms. Lecture Notes in Control and Information Science Vol. 412, Fridman L, Moreno J, Iriarte R (eds.), Springer Verlag: Berlin, 2011; 115-149.

[24] Moreno J, Osorio M. Strict Lyapunov functions for the super-twisting algorithm. IEEE Transactions on Automatic Control 2012; 57:1035-1040.

[25] Gonzalez T, Moreno J, Fridman L. Variable gain super-twisting sliding mode control. IEEE Transactions on Automatic Control 2012; 57:21002105.

[26] Zubov V. Methods of A.M. Lyapunov and Their Applications. Noordhoff, Groningen, 1964.

[27] Roxin E. On stability in control systems. J. SIAM Control 1966; 3:357-372.

[28] Clarke F, Ledyaev YS, Stern R, Wolenski P. Qualitative properties of trajectories of control systems: A survey. Journal of Dynamical and Control Systems 1995; 1(1):1-48.

[29] Chellaboina V, Leonessa A, Haddad W. Generalized Lyapunov and invariant set theorems for nonlinear dynamical systems. Systems \& Control Letters 1999; 38:289-295.

[30] Polyakov A. Nonlinear feedback design for fixed-time stabilization of linear control systems. IEEE Transactions on Automatic Control 2012; $\mathbf{5 7}(99): 2106-2110$. 
[31] Andrieu A, Praly L, Astolfi A. Homogeneous approximation, recursive obsertver and output feedback. SIAM J. Control Optim. 2008; 47:1814-1850.

[32] Cruz-Zavala E, Moreno J, Fridman L. Uniform robust exact differentiator. IEEE Transactions on Automatic Control 2011; 56(11):2727-2733.

[33] Coddington EA, Levinson N. Theory of Ordinary Differential Equations. New York, McGraw-Hill, 1955.

[34] Liberzon D. Switchings in Systems and Control. Birkhauser, Boston, 2003.

[35] Gelig AK, Leonov GA, Yakubovich VA. Stability Of Stationary Sets In Control Systems With Discontinuous Nonlinearities. World Scientific, 2004.

[36] Fridman L. An averaging approach to chattering. IEEE Transactions on Automatic Control 2001; 46:1260-1265.

[37] Zolezzi T. Differential inclusions and sliding mode control. Sliding Mode Control in Engineering, Perruquetti W, Barbot J (eds.), Marcel Dekker, $2002 ; 29-52$.

[38] Levant A. Quasi-continuous high-order sliding-mode controllers. IEEE Transactions on Automatic Control 2005; 50(11):1812-1816.

[39] Filippov AF. On certain questions in the theory of optimal control. J. SIAM Control 1962; 1(1):76-84.

[40] Poznyak A. Advanced Mathematical Tools for Automatic Control Engineers. Volume 1: Deterministic Technique. Elsevier, 2008.

[41] Gelig AK, Leonov GA, Yakubovich VA. Stability of Nonlinear Systems with Nonunique Equilibrium Position. Nauka, 1978 (in Russian).

[42] Vinograd RE. The inadequacy of the method of characteristic exponents for the study of nonlinear differential equations. Math. Sbornik 1957; 41(83):431-438. 
[43] Hahn W. Stability of Motion. Springer-Verlag Berlin Heidelberg: New York, 1967.

[44] Clarke FH, Ledyaev YS, Stern R. Asymptotic stability and smooth Lyapunov functions. Journal of differential Equations 1998; 149:69-114.

[45] Clarke F, Ledyaev YS, Stern R, Wolenski P. Nonsmooth Analysis and Control Theory. Springer, New York, 1998.

[46] Natanson I. Theory of functions of a real variable. Frederick Ungar Publishing Co., New York, 1955.

[47] Szarski J. Differential Inequalities. Polish Scientific Publishers: Warsaw, 1965.

[48] Bruckner A. Differentiation of Real Functions. Springer Verlag: Berlin, Heidelberg, New York, 1978.

[49] Aubin JP, Ekeland I. Applied Nonlinear Analysis. Wiley, New York, 1984.

[50] Clarke F. Optimization and Nonsmooth Analysis. SIAM, Philadelphia, 1990.

[51] Rademacher H. Uber partielle und totale differenzierbarkeit von funktionen mehrerer variabeln und uber die transformation der doppelintegrale. Mathematische Annalen 1919; 79:340-359.

[52] Moreau J, Valadier M. A chain rule involving vector functions of bounded variation. Journal of Functional Analysis 1987; 74(2):333-345.

[53] Wheeden R, Zygmund A. Measure and Integral: An Introduction to Real Analysis. Marcel Dekker, 1977.

[54] Polyakov A, Poznyak A. Unified Lyapunov function for a finite-time stability analysis of relay second-order sliding mode control systems. IMA Journal of Mathematical Control and Information 2012; 29(4):529-550. 
[55] Levant A. Sliding order and sliding accuracy in sliding mode control. International Journal of Control 1993; 58(6):1247-1263.

[56] Clarke FH, Vinter RB. Stability analysis of sliding-mode feedback control. Control and Cybernetics 2009; 38(4A):1169-1192.

[57] Zolezzi T. Real states of stable sliding mode control systems. Systems $\mathcal{E}$ Control Letters 2008; 57(9):778-783.

[58] Bernuau E, Polyakov A, Efimov D, Perruquetti W. Robustness of finitetime stability property for sliding modes. 5th Symposium on System Structure and Control, 2013; 391-396. 